\title{
Trace Reconstruction: Generalized and Parameterized
}

\author{
Akshay Krishnamurthy \\ College of Information and Computer Sciences, University of Massachusetts, Amherst, USA \\ akshay@cs.umass.edu
}

Arya Mazumdar

College of Information and Computer Sciences, University of Massachusetts, Amherst, USA

arya@cs.umass.edu

\author{
Andrew McGregor \\ College of Information and Computer Sciences, University of Massachusetts, Amherst, USA \\ mcgregor@cs.umass.edu
}

\section{Soumyabrata Pal}

College of Information and Computer Sciences, University of Massachusetts, Amherst, USA spal@cs.umass.edu

\begin{abstract}
In the beautifully simple-to-state problem of trace reconstruction, the goal is to reconstruct an unknown binary string $x$ given random "traces" of $x$ where each trace is generated by deleting each coordinate of $x$ independently with probability $p<1$. The problem is well studied both when the unknown string is arbitrary and when it is chosen uniformly at random. For both settings, there is still an exponential gap between upper and lower sample complexity bounds and our understanding of the problem is still surprisingly limited. In this paper, we consider natural parameterizations and generalizations of this problem in an effort to attain a deeper and more comprehensive understanding. Perhaps our most surprising results are:
\end{abstract}

1. We prove that $\exp \left(O\left(n^{1 / 4} \sqrt{\log n}\right)\right)$ traces suffice for reconstructing arbitrary matrices. In the matrix version of the problem, each row and column of an unknown $\sqrt{n} \times \sqrt{n}$ matrix is deleted independently with probability $p$. Our results contrasts with the best known results for sequence reconstruction where the best known upper bound is $\exp \left(O\left(n^{1 / 3}\right)\right)$.

2. An optimal result for random matrix reconstruction: we show that $\Theta(\log n)$ traces are necessary and sufficient. This is in contrast to the problem for random sequences where there is a superlogarithmic lower bound and the best known upper bound is $\exp \left(O\left(\log ^{1 / 3} n\right)\right)$.

3. We show that $\exp \left(O\left(k^{1 / 3} \log ^{2 / 3} n\right)\right)$ traces suffice to reconstruct $k$-sparse strings, providing an improvement over the best known sequence reconstruction results when $k=o\left(n / \log ^{2} n\right)$.

4. We show that $\operatorname{poly}(n)$ traces suffice if $x$ is $k$-sparse and we additionally have a "separation" promise, specifically that the indices of 1 's in $x$ all differ by $\Omega(k \log n)$.

2012 ACM Subject Classification Mathematics of computing $\rightarrow$ Probability and statistics

Keywords and phrases deletion channel, trace reconstruction, matrix reconstruction

Digital Object Identifier 10.4230/LIPIcs.ESA.2019.68

Funding Akshay Krishnamurthy: Supported in part by NSF Award 1763618.

Arya Mazumdar: Supported in part by NSF Awards 1642658 and 1642550.

Andrew McGregor: Supported in part by NSF Award 1637536.

Soumyabrata Pal: Supported in part by NSF Awards 1642658 and 1642550.

\footnotetext{
(c) (i) () Akshay Krishnamurthy, Arya Mazumdar, Andrew McGregor, and Soumyabrata Pal; licensed under Creative Commons License CC-BY

27th Annual European Symposium on Algorithms (ESA 2019).

Editors: Michael A. Bender, Ola Svensson, and Grzegorz Herman; Article No. 68; pp. 68:1-68:25

Leibniz International Proceedings in Informatics

LI I ICS Schloss Dagstuhl - Leibniz-Zentrum für Informatik, Dagstuhl Publishing, Germany
} 


\section{Introduction}

In the trace reconstruction problem, first proposed by Batu et al. [4], the goal is to reconstruct an unknown string $x \in\{0,1\}^{n}$ given a set of random subsequences of $x$. Each subsequence, or "trace", is generated by passing $x$ through the deletion channel in which each entry of $x$ is deleted independently with probability $p$. The locations of the deletions are not known; if they were, the channel would be an erasure channel. The central question is to find how many traces are required to exactly reconstruct $x$ with high probability.

This intriguing problem has attracted significant attention from a large number of researchers $[4,8,10,11,15,17,18,21,24,26-28]$. In a recent breakthrough, De et al. [11] and Nazarov and Peres [26] independently showed that $\exp \left(O\left((n / q)^{1 / 3}\right)\right)$ traces suffice where $q=1-p$. This bound is achieved by a mean-based algorithm, which means that the only information used is the fraction of traces that have a 1 in each position. While $\exp \left(O\left((n / q)^{1 / 3}\right)\right)$ is known to be optimal amongst mean-based algorithms, the best algorithmindependent lower bound is the much weaker $\Omega\left(n^{5 / 4} / \log n\right)[16]$.

Many variants of the problem have also been considered including: (1) larger alphabets and (2) an average case analysis where $x$ is drawn uniformly from $\{0,1\}^{n}$. Larger alphabets are only easier than the binary case, since we can encode the alphabet in binary, e.g., by mapping a single character to 1 and the rest to 0 and repeating for all characters. In the average case analysis, the state-of-the-art result is that $\exp \left(O\left(\log ^{1 / 3}(n)\right)\right)$ traces suffice ${ }^{1}$, whereas $\Omega\left(\log ^{9 / 4} n / \sqrt{\log \log n}\right)$ traces are necessary [15-17]. Very recently, and concurrent with our work, other variants have been studied including a) where the bits of $x$ are associated with nodes of a tree whose topology determines the distribution of traces generated [10] and b) where $x$ is a codeword from a code with $o(n)$ redundancy [8].

In order to develop a deeper understanding of this intriguing problem, we consider finegrained parameterization and structured generalizations of trace reconstruction. We prove several new results for these variations that shed new light on the problem. Moreover, in studying these settings, we refine existing tools and introduce new techniques that we believe may be helpful in closing the gaps in the fully general problem.

\section{$1.1 \quad$ Our Results}

Parametrizations. We begin by considering parameterizations of the trace reconstruction problem. Given the important role that sparsity plays in other reconstruction problems (see, e.g., Gilbert and Indyk [13]), we first study the recovery of sparse strings. Here we prove the following result.

- Theorem 1. If $x$ has at most $k$ non-zeros, $\exp \left(O\left((k / q)^{1 / 3} \log ^{2 / 3} n\right)\right)$ traces suffice to recover $x$ exactly, with high probability, where $q=1-p=\Omega\left(k^{-1 / 2} \log ^{1 / 2} n\right)$ is the retention probability.

As some points of comparison, note that there is a trivial $\exp (O(k / q+\log n))$ upper bound, which our result improves on with a polynomially better dependence on $k / q$ in the exponent. The best known results for the general case is $\exp \left(O\left((n / q)^{1 / 3}\right)\right)[11,26]$ and our result is a strict improvement when $k=o\left(n / \log ^{2} n\right)$. Note that since we have no restrictions on $k$ in the statement, improving upon $\exp \left(O\left((k / q)^{1 / 3}\right)\right)$ would imply an improved bound in the general setting.

\footnotetext{
${ }^{1} p$ is assumed to be constant in that work.
} 
Somewhat surprisingly, our actual result is considerably stronger (See Corollary 7 for a precise statement). We also obtain $\exp \left(O\left((k / q)^{1 / 3} \log ^{2 / 3} n\right)\right)$ sample complexity in an asymmetric deletion channel, where each 0 is deleted with probability exponentially close to 1 , but each 1 is deleted with probability $p=1-q$. With such a channel, all but a vanishingly small fraction of the traces contain only 1s, yet we are still able to exactly identify the location of every 0 . Since we can accommodate $k=\Theta(n)$ this result also applies to the general case with an asymmetric channel, yielding improvements over De et al. [11] and Nazarov and Peres [26].

We elaborate more on our techniques in the next section, but the result is obtained by establishing a connection between trace reconstruction and learning binomial mixtures. There is a large body of work devoted to learning mixtures [1,2,5, 7, 9, 12, 14, 19, 20, 25] where it is common to assume that the mixture components are well-separated. In our context, separation corresponds to a promise that each pair of 1s in the original string is separated by a 0 -run of a certain length. Our second result concerns strings with a separation promise.

- Theorem 2. If $x$ has at most $k 1$ s and each 1 is separated by 0-run of length $\Omega(k \log n)$, then, with $p=1 / 2$, $\operatorname{poly}(n)$ traces suffice to recover $x$ with high probability.

Note that reconstruction with poly $(n)$ traces is straightforward if every 1 is separated by a 0-run of length $\Omega(\sqrt{n \log n})$; the basic idea is that we can identify which $1 \mathrm{~s}$ in a collection of traces correspond to the same 1 in the original sequence and then we can use the indices of these $1 \mathrm{~s}$ in their respective traces to infer the index of the 1 in the original string. However, reducing to $\Omega(k \log n)$ separation is rather involved and is perhaps the most technically challenging result in this paper.

Here as well, we actually obtain a slightly stronger result. Instead of parameterizing by the sparsity and the separation, we instead parameterize by the number of runs, and the run lengths, where a run is a contiguous sequence of the same character. We require that each 0-run has length $\Omega(r \log n)$, where $r$ is the total number of runs. Note that this parameterization yields a stronger result since $r$ is at most $2 k+1$ if the string is $k$ sparse, but it can be much smaller, for example if the 1-runs are very long. On the other hand, the best lower bound, which is $\Omega\left(n^{5 / 4} / \log n\right)$ [16], considers strings with $\Omega(n)$ runs and run length $O(1)$.

As our last parametrization, we consider a sparse testing problem. We specifically consider testing whether the true string is $x$ or $y$, with the promise that the Hamming distance between $x$ and $y, \Delta(x, y)$, is at most $2 k$. This question is naturally related to sparse reconstruction, since the difference sequence $x-y \in\{-1,0,1\}^{n}$ is $2 k$ sparse, although of course neither string may be sparse on its own. Here we obtain the following result.

- Theorem 3. For any pair $x, y \in\{0,1\}^{n}$ with $\Delta(x, y) \leq 2 k$, $\exp (O(k \log n))$ traces suffice to distinguish between $x$ and $y$ with high probability.

Generalizations. Turning to generalizations, we consider a natural multivariate version of the trace reconstruction problem, which we call matrix reconstruction. Here we receive matrix traces of an unknown binary matrix $X \in\{0,1\}^{\sqrt{n}} \times \sqrt{n}$, where each matrix trace is obtained by deleting each row and each column with probability $p$, independently. Here the deletion channel is much more structured, as there are only $2 \sqrt{n}$ random bits, rather than $n$ in the sequence case. Our results show that we can exploit this structure to obtain improved sample complexity guarantees.

In the worst case, we prove the following theorem. 
- Theorem 4. For the matrix deletion channel with deletion probability $p$,

$$
\exp \left(O\left(n^{1 / 4} \sqrt{p \log n} / q\right)\right)
$$

traces suffice to recover an arbitrary matrix $X \in\{0,1\}^{\sqrt{n} \times \sqrt{n}}$.

While no existing results are directly comparable, it is possible to obtain $\exp \left(O\left(n^{1 / 3} \log n\right)\right)$ sample complexity via a combinatorial result due to Kós et al. [22]. This agrees with the results from the sequence case, but is obtained using very different techniques. Additionally, our proof is constructive, and the algorithm is actually mean-based, so the only information it requires are estimates of the probabilities that each received entry is 1 . As we mentioned, for the sequence case, both Nazarov and Peres [26] and De et al. [11] prove a $\exp \left(\Omega\left(n^{1 / 3}\right)\right)$ lower bound for mean-based algorithms. Thus, our result provides a strict separation between matrix and sequence reconstruction, at least from the perspective of mean-based approaches.

Lastly, we consider the random matrix case, where every entry of $X$ is drawn iid from $\operatorname{Ber}(1 / 2)$. Here we show that $O(\log n)$ traces are sufficient.

- Theorem 5. For any constant deletion probability $p<1, O(\log n)$ traces suffice to reconstruct a random $X \in\{0,1\}^{\sqrt{n} \times \sqrt{n}}$ with high probability over the randomness in $X$ and the channel.

This result is optimal, since with $o(\log n)$ traces, there is reasonable probability that a row/column will be deleted from all traces, at which point recovering this row/column is impossible. The result should be contrasted with the analogous results in the sequence case. For sequences, the best results for random strings is $\exp \left(O\left(\log ^{1 / 3} n\right)\right)$ [17] and $\Omega\left(\log ^{9 / 4} n / \sqrt{\log \log n}\right)$ [16]. In light of the lower bound for sequences, it is suprising that matrix reconstruction admits $O(\log n)$ sample complexity.

\subsection{Our Techniques}

To prove our results, we introduce several new techniques in addition to refining and extending many existing ideas in prior trace reconstruction results.

Theorem 1 is proved via a reduction from trace reconstruction to learning the parameters of a mixture of binomial distributions. Surprisingly, this natural connection does not seem to have been observed in the earlier literature. We then use a generalization of a complex-analytic approach introduced by De et al. [11] and Nazarov and Peres [26] to prove a bound on the sample complexity of learning a binomial mixture. This generalization is to move beyond the analysis of Littlewood polynomials, i.e., polynomials with $\{-1,0,1\}$ coefficients, to the case where coefficients have bounded precision. The generalization is not difficult. This is our simplest result to prove but we consider the final result to be revealing as it shows that sparsity plays a more important role than length in the complexity of trace reconstruction.

Our most technically involved result is Theorem 2 . This is proved via an algorithm that constructs a hierarchical clustering of the individual 1s in all received traces according to their corresponding position in the original string. This clustering step requires a careful recursion, where in each step we ensure no false negatives (two 1s from the same origin are always clustered together) but we have many false positives, which we successively reduce. At the bottom of the recursion, we can identify a large fraction 1s from each 1 in the original string. However, as the recursion eliminates many of the 1s, simply averaging the positions of the surviving fraction leads to a biased estimate. To resolve this, we introduce a de-biasing step which eliminates even more 1s, but ensures the survivors are unbiased, so that we can accurately estimate the location of each 1 in the original string. The initial recursion has $L=\log \log n$ levels, which is critical since the debiasing step involves conditioning on the presence of $2^{L} 1 \mathrm{~s}$ in a trace, which only happens with probability $2^{-2^{L}}=1 / n$. 
Theorem 3 leverages combinatorial arguments about $k$-decks (the multiset of subsequences of a string) due to Krasikov and Roditty [23]. The result demonstrates the utility of these combinatorial tools in trace reconstruction. As further evidence for the utility of combinatorial tools, the connection to $k$-decks was also used by Ban et al. [3] in independent concurrent work on the deletion channel.

For Theorem 4, we return to the complex-analytic approach and extend the Littlewood polynomial argument to multivariate polynomials. Since the unknown matrices are $\sqrt{n} \times \sqrt{n}$, we can use a natural bivariate polynomial of degree $O(\sqrt{n})$, which yields the improvement. However, the result of Borwein and Erdélyi [6] used in previous work on trace reconstruction applies only to univariate polynomials. Our key technical result is a generalization of their result to accommodate bivariate Littlewood polynomials, which we then use to demonstrate separation.

For Theorem 5, using an averaging argument and exploiting randomness in the original matrix, we construct a statistical test to determine if two rows (or columns) from two different traces correspond to the same row (column) in the original string. We show that this test succeeds with overwhelming probability, which lets us align the rows and columns in all traces. Once aligned, we know which rows/columns were deleted from each trace, so we can simply read off the original matrix $X$.

Notation. Throughout, $n$ is the length of the binary string being reconstructed, $n_{0}$ is the number of $0 \mathrm{~s}, k$ is the number of 1 s, i.e., the sparsity or weight. For matrices $n$ is the total number of entries, and we focus on square $\sqrt{n} \times \sqrt{n}$ matrices. For most of our results, we assume that $n, n_{0}, k$ are known since, if not, they can easily be estimated using a polynomial number of traces. Let $p$ denote the deletion probability when the 1 s and os are deleted with the same probability. We also study a channel where the 1 s and 0 s are deleted with different probabilities; in this case, $p_{0}$ is the deletion probability of a 0 and $p_{1}$ is the deletion probability of a 1 . We refer to the corresponding channel as the $\left(p_{0}, p_{1}\right)$-Deletion Channel or the asymmetric deletion channel. It will also be convenient to define $q=1-p, q_{0}=1-p_{0}$ and $q_{1}=1-p_{1}$ as the corresponding retention probabilities. Throughout, $m$ denotes the number of traces.

\section{Sparsity and Learning Binomial Mixtures}

We begin with the sparse trace reconstruction problem, where we assume that the unknown string $x$ has at most $k$ 1s. Our analysis for this setting is based on a simple reduction from trace reconstruction to learning a mixture of binomial distributions, followed by a new sample complexity guarantee for the latter problem. This approach yields two new results: first, we obtain an $\exp \left(O\left(\left(k / q_{1}\right)^{1 / 3} \log ^{2 / 3} n\right)\right)$ sample complexity bound for sparse trace reconstruction, and second, we show that this guarantee applies even if the deletion probability for 0 s is exponentially close to 1 .

To establish our results, we introduce a slightly more challenging channel which we refer to as the Austere Deletion Channel. The bulk of the proof analyzes this channel, and we obtain results for the $\left(p_{0}, p_{1}\right)$ channel via a simple reduction.

- Theorem 6 (Austere Deletion Channel Reconstruction). In the Austere Deletion Channel, all but exactly one 0 are deleted (the choice of which 0 to retain is made uniformly at random) and each 1 is deleted with probability $p_{1}$. For such a channel,

$$
m=\exp \left(O\left(\left(k / q_{1}\right)^{1 / 3} \log ^{2 / 3} n\right)\right)
$$

traces suffice for sparse trace reconstruction where $q_{1}=1-p_{1}$, provided $q_{1}=\Omega\left(\sqrt{k^{-1} \log n}\right)$. 
We will prove this result shortly, but we first derive our main result for this section as a simple corollary.

- Corollary 7 (Deletion Channel Reconstruction). For the $\left(p_{0}, p_{1}\right)$-deletion channel,

$$
m=q_{0}^{-1} \exp \left(O\left(\left(k / q_{1}\right)^{1 / 3} \log ^{2 / 3} n\right)\right)
$$

traces suffice for sparse trace reconstruction where $q_{0}=1-p_{0}$ and $q_{1}=1-p_{1}=$ $\Omega\left(\sqrt{k^{-1} \log n}\right)$.

Proof. This follows from Theorem 6. By focusing on just a single 0 , it is clear that the probability that a trace from the $\left(p_{0}, p_{1}\right)$-deletion channel contains at least one 0 is at least $q_{0}$. If among the retained 0s we keep one at random and remove the rest, we generate a sample from the austere deletion channel. Thus, with $m$ samples from the $\left(p_{0}, p_{1}\right)$ deletion channel, we obtain at least $m q_{0}$ samples from the austere channel and the result follows. Note that Theorem 1 is a special case where $p_{0}=p_{1}=p$.

Remarks. First, note that the case where $q_{1}$ is constant (a typical setting for the problem) and $k=o(\log n)$ is not covered by the corollary. However, in this case a simpler approach applies to argue that $\operatorname{poly}(n)$ traces suffice: with probability $q_{1}^{k} \geq 1 / \operatorname{poly}(n)$ no 1 s are deleted in the generation of the trace and given $\operatorname{poly}(n)$ such traces, we can infer the original position of each 1 based on the average position of each 1 in each trace. Second, note that the weak dependence on $q_{0}$ ensures that as long as $q_{0}=1 / \exp \left(O\left(\left(k / q_{1}\right)^{1 / 3} \log ^{2 / 3} n\right)\right)$, we still have the $\exp \left(O\left(\left(k / q_{1}\right)^{1 / 3} \log ^{2 / 3} n\right)\right)$ bound. Thus, our result shows that sparse trace reconstruction is possible even when zeros are retained with exponentially small probability.

Reduction to Learning Binomial Mixtures. We prove Theorem 6 via a reduction to learning binomial mixtures. Given a string $x$ of length $n$, let $r_{i}$ be the number of ones before the $i^{\text {th }}$ zero in $x$. For example, if $x=1001100$ then $r_{1}=1, r_{2}=1, r_{3}=3, r_{4}=3$. Note that the multi-set $\left\{r_{1}, r_{2}, \ldots,\right\}$ uniquely determines $x$, that each $r_{i} \leq k$, and that the multi-set has size $n_{0}$. The reduction from trace reconstruction to learning binomial mixtures is appealingly simple:

1. Given traces $t_{1}, \ldots, t_{m}$ from the austere channel, let $s_{i}$ be the number of leading ones in $t_{i}$.

2. Observe that each $s_{i}$ is generated by a uniform ${ }^{2}$ mixture of $\operatorname{Bin}\left(r_{1}, q_{1}\right), \ldots, \operatorname{Bin}\left(r_{n_{0}}, q_{1}\right)$ where $q_{1}=1-p_{1}$. Hence, learning $r_{1}, r_{2}, \ldots, r_{n_{0}}$ from $s_{1}, s_{2}, \ldots, s_{m}$ allows us to reconstruct $x$.

To obtain Theorem 6, we establish the following new guarantee for learning binomial mixtures.

- Theorem 8 (Learning Binomial Mixtures). Let $\mathcal{M}$ be a mixture of $d=\operatorname{poly}(n)$ binomials:

Draw sample from $\operatorname{Bin}\left(a_{i}, q\right)$ with probability $\alpha_{i}$

where $0 \leq a_{1}, \ldots, a_{d} \leq a$ are distinct integers, the values $\alpha_{i}$ have $\operatorname{poly}(n)$ precision, and $q=\Omega\left(\sqrt{a^{-1} \log n}\right)$. Then $\exp \left(O\left((a / q)^{1 / 3} \log ^{2 / 3} n\right)\right)$ samples suffice to learn the parameters exactly with high probability.

${ }^{2}$ Note that since the $r_{i}$ are not necessarily distinct some of the binomial distributions are the same. 
Proof. Let $\mathcal{M}^{\prime}$ be a mixture where the samples are drawn from $\sum_{i=1}^{d} \beta_{i} \operatorname{Bin}\left(b_{i}, q\right)$, where $0 \leq b_{1}, \ldots, b_{d} \leq a$ are distinct and the probabilities $\beta_{i} \in\{0, \gamma, 2 \gamma, \ldots, 1\}$ where $1 / \gamma=\operatorname{poly}(n)$. Consider the variational distance $\sum_{i}\left|A_{i}-B_{i}\right|$ between $\mathcal{M}$ and $\mathcal{M}^{\prime}$ where

$$
\begin{array}{ll}
A_{i}= & \operatorname{Pr}[\text { sample from } \mathcal{M} \text { is } i]=\sum_{j=1}^{d} \alpha_{j}\left(\begin{array}{c}
a_{j} \\
i
\end{array}\right) q^{i}(1-q)^{a_{j}-i} \\
B_{i}= & \operatorname{Pr}\left[\text { sample from } \mathcal{M}^{\prime} \text { is } i\right]=\sum_{j=1}^{d} \beta_{j}\left(\begin{array}{c}
b_{j} \\
i
\end{array}\right) q^{i}(1-q)^{b_{j}-i} .
\end{array}
$$

We will show that the variational distance between $\mathcal{M}$ and $\mathcal{M}^{\prime}$ is at least

$$
\epsilon=\exp \left(-O\left((a / q)^{1 / 3}(\log 1 / \gamma)^{2 / 3}\right)\right)
$$

Since there are at most $((a+1) \cdot(1 / \gamma+1))^{d}$ possible choices for the parameters of $\mathcal{M}^{\prime}$, standard union bound arguments show that

$$
O\left(\log \left(((a+1) \cdot(1 / \gamma+1))^{d}\right) / \epsilon^{2}\right)=\exp \left(O\left((a / q)^{1 / 3}(\log 1 / \gamma)^{2 / 3}\right)\right)
$$

samples are sufficient to distinguish $\mathcal{M}$ from all other mixtures.

To prove the total variation bound, observe that by applying the binomial formula, for any complex number $w$, we have

$$
\begin{array}{rlrl}
\sum_{i \geq 0}\left(A_{i}-B_{i}\right) w^{i} & =\sum_{i \geq 0} w^{i}\left(\sum_{j \geq 0} \alpha_{j}\left(\begin{array}{c}
a_{j} \\
i
\end{array}\right) q^{i}(1-q)^{a_{j}-i}-\beta_{j}\left(\begin{array}{c}
b_{j} \\
i
\end{array}\right) q^{i}(1-q)^{b_{j}-i}\right) \\
& = & \sum_{j \geq 0}\left(\alpha_{j} z^{a_{j}}-\beta_{j} z^{b_{j}}\right)
\end{array}
$$

where $z=q w+(1-q)$. Let $G(z)=\sum_{j \geq 0}\left(\alpha_{j} z^{a_{j}}-\beta_{j} z^{b_{j}}\right)$ and apply the triangle inequality to obtain:

$$
\sum_{i \geq 0}\left|A_{i}-B_{i}\right|\left|w^{i}\right| \geq|G(z)|
$$

Note that $G(z)$ is a non-zero degree $d$ polynomial with coefficients in the set

$$
\{-1, \ldots,-2 \gamma,-\gamma, 0, \gamma, 2 \gamma, \ldots, 1\} \text {. }
$$

We would like to find a $z$ such that $G(z)$ has large modulus but $\left|w^{i}\right|$ is small, since this will yield a total variation lower bound. We proceed along similar lines to Nazarov and Peres [26] and De et al. [11]. It follows from Corollary 3.2 in Borwein and Erdélyi [6] that there exists $z \in\left\{e^{i \theta}:-\pi / L \leq \theta \leq \pi / L\right\}$ such that

$$
|G(z)| \geq \gamma \exp \left(-c_{1} L \log (1 / \gamma)\right)
$$

for some constant $c_{1}>0$. For such a value of $z$, Nazarov and Peres [26] show that

$$
|w| \leq \exp \left(c_{2} /(q L)^{2}\right)
$$

for some constant $c_{2}>0$. Therefore,

$$
\sum_{i \geq 0}\left|A_{i}-B_{i}\right| \exp \left(i c_{2} /(q L)^{2}\right) \geq \sum_{i \geq 0}\left|A_{i}-B_{i}\right|\left|w^{i}\right| \geq|G(z)| \geq \gamma \exp \left(-c_{1} L \log (1 / \gamma)\right)
$$


For $i>\tau=6 q a$, by an application of the Chernoff bound, $A_{i}, B_{i} \leq 2^{-i}$, so we obtain

$$
\underbrace{\sum_{i>\tau} 2^{-i} \exp \left(i c_{2} /(q L)^{2}\right)}_{=T_{\tau}}+\sum_{i=0}^{\tau}\left|A_{i}-B_{i}\right| \exp \left(\tau c_{2} /(q L)^{2}\right) \geq \gamma \exp \left(-c_{1} L \log (1 / \gamma)\right) .
$$$$
\sum_{i=0}^{\tau}\left|A_{i}-B_{i}\right| \geq \frac{\exp \left(-c_{1} L \log (1 / \gamma)\right)}{\exp \left(\tau c_{2} /(q L)^{2}\right)}-\frac{T_{\tau}}{\exp \left(\tau c_{2} /(q L)^{2}\right)} \geq \frac{\gamma \exp \left(-c_{1} L \log (1 / \gamma)\right)}{\exp \left(\tau c_{2} /(q L)^{2}\right)}-O\left(2^{-\tau}\right)
$$

where the second equality follows from the assumption that $c_{2} /\left(q L^{2}\right) \leq(\ln 2) / 2$ (which we will ensure when we set $L$ ) since,

$$
\frac{T_{\tau}}{\exp \left(\tau c_{2} /(q L)^{2}\right)}=\frac{O(1) \cdot 2^{-\tau} \exp \left(\tau c_{2} /(q L)^{2}\right)}{\exp \left(\tau c_{2} /(q L)^{2}\right)}=O\left(2^{-\tau}\right) .
$$

Set

$$
L=c \sqrt[3]{\tau /\left(q^{2} \log (1 / \gamma)\right)}=c \sqrt[3]{6 a /(q \log (1 / \gamma))}
$$

for some sufficiently large constant $c$. This ensures that the first term of Eqn. 1 is

$$
\exp \left(-O\left((a / q)^{1 / 3} \log ^{2 / 3}(1 / \gamma)\right)\right) .
$$

Note that

$$
\frac{c_{2}}{q L^{2}}<\frac{c_{2}}{q c^{2}(a /(q \log (1 / \gamma)))^{2 / 3}} \leq \frac{c_{2}}{c^{2}} \cdot\left(\frac{\log (1 / \gamma)}{a q^{1 / 2}}\right)^{2 / 3} \leq \frac{c_{2}}{c^{2}} \cdot\left(\frac{\log (1 / \gamma)}{a q^{2}}\right)^{2 / 3}
$$

and so by the assumption that $q=\Omega(\sqrt{\log (1 / \gamma) / a})$ we may set the constant $c$ large enough such that $c_{2} /\left(q L^{2}\right) \leq(\ln 2) / 2$ as required. The second term of Eqn. 1 is a lower order term given the assumption from the assumption on $q$ and thus we obtain the required lower bound on the total variation distance.

Theorem 6 now follows from Theorem 8, since in the reduction, we have $d=O(n)$ binomials, one per 0 in $x, \alpha_{i}$ is a multiple of $1 / n_{0}$ and importantly, we have $a=k$. The key is that we have a polynomial with degree $a=k$ rather than a degree $n$ polynomial as in the previous analysis.

Remark. If all $\alpha_{i}$ are equal, Theorem 8 can be improved to $\operatorname{poly}(n) \cdot \exp \left(O\left((a / p)^{1 / 3}\right)\right)$ by using a more refined bound from Borwein and Erdélyi [6] in our proof. This follows by observing that if $\alpha_{i}=\beta_{i}=1 / d$, then $\sum_{j \geq 0}\left(\alpha_{j} z^{a_{j}}-\beta_{j} z^{s_{j}}\right)$ is a multiple of a Littlewood polynomial and we may use the stronger bound $|G(z)| \geq \exp \left(-c_{1} L\right) / d$, see Borwein and Erdélyi [6].We can also show that the exponential dependence on $a^{1 / 3}$ in Theorem 8 is necessary.

- Theorem 9 (Binomial Mixtures Lower Bound). There exists subsets

$$
\left\{a_{1}, \ldots, a_{k}\right\} \neq\left\{b_{1}, \ldots, b_{k}\right\} \subset\{0, \ldots, a\}
$$

such that if $\mathcal{M}=\sum_{i=1}^{k} \operatorname{Bin}\left(a_{i}, 1 / 2\right) / k$ and $\mathcal{M}^{\prime}=\sum_{i=1}^{k} \operatorname{Bin}\left(b_{i}, 1 / 2\right) / k$, then $\left\|\mathcal{M}-\mathcal{M}^{\prime}\right\|_{T V}=$ $\exp \left(-\Omega\left(a^{1 / 3}\right)\right)$. Thus, $\exp \left(\Omega\left(a^{1 / 3}\right)\right)$ samples are required to distinguish $\mathcal{M}$ from $\mathcal{M}^{\prime}$. 
Proof. Previous work $[11,26]$ shows the existence of two strings $x, y \in\{0,1\}^{n}$ such that $\sum_{i}\left|t_{i}^{x}-t_{i}^{y}\right|=\exp \left(-\Omega\left(n^{1 / 3}\right)\right)$ where $t_{i}^{z}$ is the expected value of the $i$ th element (indexed at 0$)$ of a string formed applying the $(1 / 2,1 / 2)$-deletion channel to the string $z$. We may assume $\sum_{i \in[n]} x_{i}=\sum_{i \in[n]} y_{i} \equiv k$ since otherwise

$$
\sum_{i}\left|t_{i}^{x}-t_{i}^{y}\right| \geq\left|\sum_{i} t_{i}^{x}-\sum_{i} t_{i}^{y}\right|=\left|\sum_{i \in[n]} x_{i} / 2-\sum_{i \in[n]} y_{i} / 2\right| \geq 1 / 2
$$

which would contradict the assumption $\sum_{i}\left|t_{i}^{x}-t_{i}^{y}\right| \neq \exp \left(-\Omega\left(n^{1 / 3}\right)\right)$.

Consider $\mathcal{M}=\sum_{i=1}^{k} \operatorname{Bin}\left(a_{i}, 1 / 2\right) / k$ and $\mathcal{M}^{\prime}=\sum_{i=1}^{k} \operatorname{Bin}\left(b_{i}, 1 / 2\right) / k$, where $a_{i}\left(b_{i}\right)$ is the number of coordinates preceding the $i$ th 1 in $x(y)$. Note that

$$
t_{i}^{x}=\sum_{r=1}^{k}\left(\begin{array}{c}
a_{r} \\
i
\end{array}\right) / 2^{a_{r}+1} \quad \text { and } \quad t_{i}^{y}=\sum_{r=1}^{k}\left(\begin{array}{c}
b_{r} \\
i
\end{array}\right) / 2^{b_{r}+1},
$$

and so

$$
\begin{aligned}
\left\|\mathcal{M}-\mathcal{M}^{\prime}\right\|_{T V} & = & \sum_{i}\left|\operatorname{Pr}[\mathcal{M}=i]-\operatorname{Pr}\left[\mathcal{M}^{\prime}=i\right]\right| \\
& = & \sum_{i} \frac{1}{k}\left|\sum_{r=1}^{k}\left(\begin{array}{c}
a_{r} \\
i
\end{array}\right) / 2^{a_{r}}-\sum_{r=1}^{k}\left(\begin{array}{c}
b_{r} \\
i
\end{array}\right) / 2^{b_{r}}\right| \\
& = & \frac{2}{k} \sum_{i}\left|t_{i}^{x}-t_{i}^{y}\right|=\exp \left(-\Omega\left(n^{1 / 3}\right)\right),
\end{aligned}
$$

which proves the result.

\section{Well-Separated Sequences}

We now prove Theorem 2, showing that $\operatorname{poly}(n)$ traces suffice for reconstruction of a $k$-sparse string when there are $\Omega(k \log n)$ 0s between each consecutive 1 . We call such sequences of 0 s the 0 -runs of the string. We also refer to the length of the shortest 0-run as the gap $g$ of the string $x$.

- Theorem (Restatement of Theorem 2). Let $x$ be a k-sparse string of length $n$ and gap at least ck $\log (n)$ for a large enough $c$. Then $\operatorname{poly}(n)$ traces from the $(1 / 2,1 / 2)$-Deletion Channel suffice to recover $x$ with high probability.

In Section 3.1, we present the basic ideas and technical challenges in proving the theorem. We also describe the algorithm in detail and explain how to set the parameters. Full details are presented in Section 3.3. In Section 3.2, we strengthen Theorem 2 to show that poly $(n)$ traces suffice under the weaker assumption that each 0-run has length $\tilde{\Omega}(r)$ where $r$ is the total number of runs (0-runs +1 -runs). Observe that this is a weaker assumption, since $r \leq 2 k+1$ always, but $r$ can be much less than $k$.

\subsection{A Recursive Hierarchical Clustering Algorithm and Its Analysis: Overview}

Let $\left\{p_{u}\right\}_{u=1}^{k}$ denote the positions (index of the coordinate from the left) of the $k$ is in the original string $x$. Let $\mathcal{N}$ denote the multi-set of all positions of all received $1 \mathrm{~s}$ and call $N=|\mathcal{N}|$. We will construct a graph $G$ on $N$ vertices where every vertex is associated with a 
received 1 . We decorate each vertex $v$ with a number $z_{v} \in \mathcal{N}$, which is the position of the associated received 1. Each vertex $v$ also has an unknown label $y_{v} \in\{1, \ldots, k\}$ denoting the corresponding 1 in the original string.

At a high level, our approach uses the observed values $\left\{z_{v}\right\}_{v \in V}$ to recover the unknown labels $\left\{y_{v}\right\}_{v \in V}$. Once this "alignment" has been performed, the original string can be recovered easily, since the average of $\left\{z_{v} \mathbf{1}\left\{y_{v}=u\right\}\right\}_{v \in V}$ is an unbiased estimator for $p_{u} / 2$.

A starting observation. Our first observation is a simple fact about binomial concentration, which we will use to define the edge set in $G$ : by the Chernoff bound, with high probability, for every vertex $v$, if $y_{v}=u$ then we must have $\left|z_{v}-p_{u} / 2\right| \leq c \sqrt{n \log n}$ for some constant $c$. Defining the edges in $G$ to be $\left\{(v, w):\left|z_{v}-z_{w}\right| \leq 2 c \sqrt{n \log n}\right\}$ then guarantees that all vertices with $y_{v}=u$ are connected. This immediately yields an algorithm for the much stronger gap condition $g \geq 4 c \sqrt{n \log n}$, since with such separation, no two vertices $v, w$ with $y_{v} \neq y_{w}$ will have an edge. Therefore, the connected components reveal the labeling so that $\operatorname{poly}(n)$ traces suffice with $g=\Omega(\sqrt{n \log n})$.

Intuitively, we have constructed a clustering of the received 1s that corresponds to the underlying labeling. To tolerate a weaker gap condition, we proceed recursively, in effect constructing a hierarchical clustering. However there are many subtleties that must be resolved.

The first recursion. To proceed, let us consider the weaker gap condition of $g \geq \tilde{\Omega}\left(k^{1 / 2} n^{1 / 4}\right)$. In this regime, $G$ still maintains a consistency property that for each $u$ all vertices with $y_{v}=u$ are in the same connected component, but now a connected component may have vertices with different labels, so that each connected component $C$ identifies a continguous set $U \subset\{1, \ldots, k\}$ of the original 1s. Moreover, due to the sparsity assumption, $C$ must have length, defined as $\max _{v \in C} z_{v}-\min _{v \in C} z_{v}$, at most $O(k \sqrt{n \log n})$. Therefore if we can correctly identify every trace that contains the left-most and right-most 1 in $U$, we can recurse and are left to solve a subproblem of length $O(k \sqrt{n \log n})$. Appealing to our starting observation, this can be done with a gap of $g \geq \tilde{\Omega}\left(k^{1 / 2} n^{1 / 4}\right)$.

The challenge for this step is in identifying every trace that contains the left-most and right-most 1 in $U$, which we call $u_{L}$ and $u_{R}$ respectively. This is important for ensuring a "clean" recursion, meaning that the traces used in the subproblem are generated by passing exactly the same substring through the deletion channel. To solve this problem we use a device that we call a Length Filter. For every trace, consider the subtrace that starts with the first received 1 in $U$ and ends with the last received 1 in $U$ (this subtrace can be identified using $G$ ). If the trace contains $u_{L}, u_{R}$ then the length of this subtrace is $2+\operatorname{Bin}(L-2,1 / 2)$ where $L$ is the distance between $u_{L}, u_{R}$ in the original string. On the other hand, if the subtrace does not contain both end points, then the length is $2+\operatorname{Bin}\left(L^{\prime}-2,1 / 2\right)$ where $L^{\prime} \leq L-g$. Since we know that $L \leq \tilde{O}(k \sqrt{n})$ and we are operating with gap condition $g=\tilde{\Omega}\left(k^{1 / 2} n^{1 / 4}\right)=\tilde{\Omega}(\sqrt{L})$, binomial concentration implies that with high probability we can exactly identify the subtraces containing $u_{L}$ and $u_{R}$.

Further recursion. The difficulty in applying a second recursive step is that when $g=$ $o\left(k^{1 / 2} n^{1 / 4}\right)$ the length filter cannot isolate the subtraces that contain the leftmost and rightmost 1s for a block $U$, so we cannot guarantee a clean recursion. However, substrings that pass through the filter are only missing a short prefix/suffix which upper bounds any error in the indices of the received 1s. We ensure consistency at subsequent levels by incorporating this error into a more cautious definition of the edge set (in fact the additional error is the same order as the binomial deviation at the next level, so it has negligible effect). In this 
way, we can continue the recursion until we have isolated each 1 from the original string. The $\Omega(k \log n)$ lower bound on run length arises since the gap at level $t$ of the recursion, $g_{t}$, is related to the gap at level $t-1$ via $g_{t}=\sqrt{k \log n \cdot g_{t-1}}$ with $g_{1}=\sqrt{n \log n}$, and this recursion asymptotes at $\Omega(k \log n)$.

The last technical challenge is that, while we can isolate each original 1 , the error in our length filter introduces some bias into the recursion, so simply averaging the $z_{v}$ values of the clustered vertices does not accurately estimate the original position. However, since we have isolated each 1 into pure clusters, for any connected component corresponding to a block of 1s, we can identify all traces that contain the first and last 1 in the block. Applying this idea recursively from the bottom up allows us to debias the recursion and accurately estimate all positions.

The algorithm in detail: recursive hierarchical clustering. We now describe the recursive process in more detail. Let us define the thresholds:

$$
\tau_{1}=\tilde{O}\left(n^{1 / 2}\right), \tau_{2}=\tilde{O}\left(k^{1 / 2} n^{1 / 4}\right), \tau_{3}=\tilde{O}\left(k^{3 / 4} n^{1 / 8}\right), \ldots, \tau_{D}=\tilde{O}\left(k^{1-1 / 2^{D}} n^{1 / 2^{D}}\right),
$$

which will be used in the length filter and in the definitions of the edge set. Observe that with $D=\log \log n$, we have $\tau_{D}=\tilde{O}(k)$. Let $\tilde{x}_{1}, \ldots, \tilde{x}_{m}$ denote the $m=\operatorname{poly}(n)$ traces. We will construct a sequence of graphs $G_{1}, G_{2}, \ldots, G_{D}$ on the vertex sets $V_{1} \supset V_{2}, \ldots, \supset V_{D}$, where each vertex $v$ corresponds to a received 1 in some trace $t_{v} \in[\mathrm{m}]$ and is decorated with its position $z_{v}$ and the unknown label $y_{v}$. The $d^{\text {th }}$ round of the algorithm is specified as follows with $z_{v}^{(1)}=z_{v}$ and $V_{1}$ as the set of all received $1 \mathrm{~s}$.

1. Define $G_{d}$ with edge set $E_{d}=\left\{(v, w): v, w \in V_{d}\right.$ and $\left.\left|z_{v}^{(d)}-z_{w}^{(d)}\right| \leq \tau_{d}\right\}$.

2. Extract $k_{d} \leq k$ connected components $C_{1}^{(d)}, \ldots, C_{k_{d}}^{(d)}$ from $G_{d}$.

3. For each connected component $C_{i}^{(d)}$, extract subtraces $\left\{\tilde{x}_{j}^{(d, i)}\right\}_{j=1}^{m}$ where $\tilde{x}_{j}^{(d, i)}$ is the substring of $\tilde{x}_{j}$ starting with the first 1 in $C_{i}$ and ending with the last 1 in $C_{i}$. Formally, with $\ell=\min \left\{z_{v}: v \in C_{i}, t_{v}=j\right\}$ and $r=\max \left\{z_{v}: v \in C_{i}, t_{v}=j\right\}$, we define $\tilde{x}_{j}^{(d, i)}=\tilde{x}_{j}[\ell, \ldots, r]$.

4. Length Filter: Define $L^{(d, i)}=\max _{j} \operatorname{len}\left(\tilde{x}_{j}^{(d, i)}\right)$. If

$$
\operatorname{len}\left(\tilde{x}_{j}^{(d, i)}\right) \leq L^{(d, i)}-\Omega\left(\sqrt{L^{(d, i)} \log \left(L^{(d, i)}\right)}\right),
$$

delete all vertices $v \in C_{i}$ with $t_{v}=j$. Let $V_{d+1}$ be all surviving vertices.

5. For $v \in V_{d+1}$, define $z_{v}^{(d+1)}=z_{v}-\min _{v^{\prime} \in C_{i}, t_{v}=t_{v^{\prime}}} z_{v^{\prime}}$.

We analyze the procedure via sequence of lemmas. The first one establishes a basic consistency property: that two 1s originating from the same source 1 are always clustered together.

Lemma 10 (Consistency). At level d let $V_{d, u}=\left\{v \in V_{d}, y_{v}=u\right\}$ for each $u \in[k]$. Then with high probability, for each $d$ and $u$ there exists some component $C_{i}^{(d)}$ at level $d$ such that $V_{d, u} \subset C_{i}^{(d)}$.

The next lemma provides a length upper bound on any component, which is important for the recursion. At a high level since we are using a threshold $\tau_{d}$ at level $d$ and the string is $k$-sparse, no connected component can span more than $k \tau_{d}$ positions.

- Lemma 11 (Length Bound). For every component $C_{i}^{(d)}$ at level d, we have $L^{(d, i)} \leq$ $2 k \tau_{d}$. Moreover if $U$ is a contiguous subsequence of $\{1, \ldots, k\}$ with $\bigcup_{u \in U} V_{d, u} \subset C_{i}^{(d)}$, then $\min _{u \in U} p_{u}-\max _{u \in U} p_{u} \leq 2 k \tau_{d}$. 
Finally we characterize the length filter.

- Lemma 12 (Length Filter). For a component $C_{i}^{(d)}$ at level $d$, let $U$ be the maximal contiguous subsequence of $\{1, \ldots, k\}$ such that $\bigcup_{u \in U} V_{d, u} \subset C_{i}^{(d)}$. Define $u_{L}=\arg \min _{u \in U} p_{u}$ and $u_{R}=\arg \max _{u \in U} p_{u}$. Then for any $v \in C_{i}^{(d)}$, if $u_{L}$ and $u_{R}$ are present in $t_{v}$, then $v$ survives to round $d+1$, that is $v \in V_{d+1}$. Moreover, for any $v \in V_{d+1}$, let $p_{\min }(v, U)$ denote the original position of the first 1 from $U$ that is also in the trace $t_{v}$. Then we have $p_{\min }(v, u)-p_{u_{L}} \leq \tilde{O}\left(\sqrt{k \tau_{d}}\right)$.

The lemmas are all interconnected and proved formally in Section 3.3. It is important that the error incurred by the length filter is $\sqrt{k \tau_{d}}=\tau_{d+1}$ which is exactly the binomial deviation at level $d+1$. Thus the threshold used to construct $G_{d+1}$ accounts for both the length filter error and the binomial deviation. This property, established in Lemma 12, is critical in the proof of Lemma 10.

For the hierarchical clustering, observe that after $D=\log \log n$ iterations, we have $\tau_{D}=\tilde{O}(k)$. With gap condition $g=\tilde{\Omega}(k)$ and applying Lemma 10, this means that the connected components at level $D$ each correspond to exactly one 1 in the original string. Moreover since the length filter preserves every trace containing the left-most and right-most 1 in the component, the probability that a subtrace passes through the length filter is at least $1 / 4$. Hence, after $\log \log n$ levels, the expected number of surviving traces in each cluster is $m / 4^{\log \log n}=m /\left(\log ^{2} n\right)$. Thus for each original $1 u \in\{1, \ldots, k\}$, our recursion identifies at least $m /\left(\log ^{2} n\right)$ vertices $v \in V_{1}$ such that $t_{v}=u$.

Removing Bias. The last step in the algorithm is to overcome bias introduced by the length filter. The de-biasing process works upward from the bottom of the recursion. Since we have isolated the vertices corresponding to each 1 in the original string, for a component $C_{i}^{(D-1)}$ at level $D-1$, we can identify all subtraces that survived to this level that contain the first and last 1 of the corresponding block $U_{i}^{(D-1)} \subset[k]$. Thus, we can eliminate all subtraces that erroneously passed this length filter.

Working upwards, consider a component $C_{i}^{(d)}$ that corresponds to a block $U_{i}^{(d)} \subset[k]$ of $1 \mathrm{~s}$ in the original string. Since we have performed further clustering, we have effectively partitioned $U_{i}^{(d)}$ into sub-blocks $U_{1}^{(d+1)}, \ldots, U_{s}^{(d+1)}$. We would like to identify exactly the subtraces that survived to level $d$ that contain the first and last 1 of $U_{i}^{(d)}$, but unfortunately this is not possible due to a weak gap condition. However, by induction, we can exactly identify all subtraces that survive to level $d$ that contain the first and last 1 of the first and last sub-block of $U_{i}^{(d)}$, namely $U_{1}^{(d+1)}$ and $U_{s}^{(d+1)}$. Thus we can de-bias the length filter at level $d$ by filtering based on a more stringent event, namely the presence of $2^{D-d}$ nodes. In total to de-bias all length filters above a particular component, we require the presence of $\sum_{d=1}^{D} 2^{D-d}=O\left(2^{D}\right)=O(\log n)$ nodes, which happens with probability $\Omega(1 / n)$. Thus we can debias with only a polynomial overhead in sample complexity. See Figure 1 for an illustration.

\subsection{Strengthening to a Parameterization by Runs}

We next parameterized the problem by the number of runs, $r=1+\left|\left\{i \in[n-1]: x_{i} \neq x_{i+1}\right\}\right|$, in the string $x$ being reconstructed. We will argue that if every 0-run has length $\tilde{\Omega}(r)$ then poly $(n)$ traces suffice. The proof is via a reduction to the $k$-sparse case in the previous sections.

Let $x^{\prime} \in\{0,1\}^{<n}$ be the string formed by replacing every run of 1 s in $x$ by a single 1. We first argue that we can reconstruct $x^{\prime}$ with high probability using poly $(n)$ traces generated by applying the $(1 / 2,1 / 2)$-Deletion Channel to $x$. We will prove this result for 
the case $r=\Omega(\log n)$ since otherwise poly $(n)$ traces is sufficient even with no gap promise. ${ }^{3}$ Observe that with $m=\operatorname{poly}(n)$ traces, if every 0-run in $x$ has length at least $c \log n$ for some sufficiently large constant $c>0$, then a bit in every 0-run of $x$ appears in every trace with high probability. Conditioned on this event, no two 1's that originally appeared in different runs of $x$ are adjacent in any trace. Next replace each run of $1 \mathrm{~s}$ in each trace with a single 1. The end result is that we generate traces that are generated as if we had deleted each 0 in $x^{\prime}$ with probability $1 / 2$ and each 1 in $x^{\prime}$ with probability $1-1 / 2^{t} \geq 1 / 2$ where $t$ is the length of the run that the 1 belonged to in $x$. This channel is not equivalent to the $(1 / 2,1 / 2)$-Deletion channel, but our analysis for the sparse case continues to hold even if the deletion probability of each 1 is different. Thus we can apply Theorem 2 to recover $x^{\prime}$, and the sparsity of $x^{\prime}$ is at most $r$. Since the algorithm identifies corresponding $1 \mathrm{~s}$ in $x^{\prime}$ in the different traces, we can then estimate the length of the 1-runs in $x$ that were collapsed to each single 1 of $x^{\prime}$ by looking at the lengths of the corresponding 1-runs in the traces of $x$ before they were collapsed.

- Theorem 13. For the $(1 / 2,1 / 2)$-Deletion Channel, $\operatorname{poly}(n)$ traces suffice if the lengths of the O-runs are $\tilde{\Omega}(r)$ where $r$ is the number of runs in $x$.

\subsection{Sparsity with Gap: Technical Details}

This section contains missing details from Section 3. Recall that we have a string $x \in\{0,1\}^{n}$ that is $k$-sparse. We further assume that each 1 in $x$ is separated by a run of $g$ os, and we refer to $g$ as the gap. Recall that we define $\left\{p_{u}\right\}_{u=1}^{k}$ as the position of the $k 1$ s in original string, where $p_{1}<p_{2}<\ldots, p_{k}$. As further notation we refer to the collection of $m=\operatorname{poly}(n)$ traces as $\mathcal{T}=\left\{\tilde{x}_{j}\right\}_{j=1}^{m}$.

\section{The first level}

As a warm up, we show an algorithm called FindPositions, that uses poly $(n)$ traces to reconstruct $x$ exactly with high probability when the gap $g=\Omega(\sqrt{n \log n})$. The algorithm returns the values $\left\{p_{u}\right\}_{u=1}^{k}$ and crucially uses a binomial mean estimator. Given $s$ samples $X_{1}, X_{2}, \ldots, X_{s}$ from a binomial distribution $\operatorname{Bin}\left(n, \frac{1}{2}\right)$ this estimator returns an estimate of $n, \hat{n}=\operatorname{round}\left(\frac{2}{s} \sum_{i=1}^{s} X_{i}\right)$, where the round function simply rounds the argument to the nearest integer. From the Hoeffding bound, it is clear that

$$
\begin{aligned}
\operatorname{Pr}(\hat{n} \neq n)=\operatorname{Pr}(|\hat{n}-n| \geq 0.5) & =\operatorname{Pr}\left(\left|\frac{1}{s} \sum_{i=1}^{s} X_{i}-\frac{n}{2}\right| \geq \frac{1}{4}\right) \\
& \leq 2 \exp \left(-\frac{s}{8 n^{2}}\right) \leq 2 \exp \left(-n^{\epsilon}\right),
\end{aligned}
$$

as long as $s=8 n^{2+\epsilon}$ for any $\epsilon>0$.

The algorithm FindPositions is displayed in Algorithm 1. Our first result of this section guarantees that with $g=\Omega(\sqrt{n \log n})$ Algorithm 1 recovers $x$ exactly with poly $(n)$ traces.

- Theorem 14. Algorithm 1 (FindPositions) successfully returns the string $x$ from $m$ traces with probability at least $1-3 n^{-2}$ as long as $m \geq \Omega\left(n^{2} \log n\right)$ and the gap $g \geq$ $4 \sqrt{2 n \log \left(n m^{3}\right)}=\Theta(\sqrt{n \log n})$.

\footnotetext{
${ }^{3}$ Specifically, if $r=O(\log n)$, with probability at least $1 / 2^{r}=1 / \operatorname{poly}(n)$ a trace also has $r$ runs. Given $\operatorname{poly}(n)$ traces with $r$ runs we can estimate each run length because we know the $i^{\text {th }}$ run in each such trace corresponds to the $i^{\text {th }}$ run in the original string.
} 
Algorithm 1 FindPositions.

Initialize: length of $x: n, m$ traces $\mathcal{T}$, gap $g>4 \sqrt{2 n \log \left(m n^{3}\right)}$.

For each received 1 , create a vertex $v$ decorated with tuple $\left(z_{v}, t_{v}\right)$ where $z_{v} \in[n]$ is the position of the received 1 and $t_{v} \in[m]$ is the index of the trace.

Create graph $G=(V, E)$ using vertex set above, and with edges:

$$
E=\left\{(v, w):\left|z_{v}-z_{w}\right| \leq \sqrt{2 n \log \left(m n^{3}\right)}\right\}
$$

Find connected components $C_{1}, \ldots, C_{k^{\prime}}$ in $G$ (If $k^{\prime} \neq k$ report failure).

For each connected component $C_{i}$, use the binomial mean estimator on $\left\{z_{v}\right\}_{v \in C_{i}}$ to estimate $\hat{p}_{i}$.

Return $\left\{\hat{p}_{i}\right\}_{i=1}^{k^{\prime}}$.

Proof. First, let us associate with each vertex $v$ an unknown label $y_{v} \in[k]$ describing the correspondence between this received 1 and a 1 in the original string. The first observation is that if $y_{v}=u$ then $z_{v} \sim \operatorname{Bin}\left(p_{u}, \frac{1}{2}\right)$ and we always have $p_{u} \leq n$. Thus, by Hoeffding's inequality and a union bound, we have

$$
\operatorname{Pr}\left[\exists v \in V:\left|z_{v}-p_{u} / 2\right|>\tau\right] \leq|V| \exp \left(-2 \tau^{2} / n\right) \leq \exp \left(\log (m k)-2 \tau^{2} / n\right)
$$

And so with $\tau=\sqrt{n \log \left(m k n^{2}\right) / 2}$, with probability $1-n^{-2}$ all $z_{v}$ values concentrate appropriately.

This event immediately implies that $G$ is consistent in the sense that if $y_{v}=y_{v^{\prime}}$ then $\left(v, v^{\prime}\right) \in E$. Further the gap condition implies the converse property, which we call purity: if $y_{v} \neq y_{v^{\prime}}$ then $\left(v, v^{\prime}\right) \notin E$. Formally, if $y_{v} \neq y_{v^{\prime}}$ then

$$
\begin{aligned}
g / 2 & \leq\left|p_{y_{v}} / 2-p_{y_{v^{\prime}}} / 2\right| \\
& \leq\left|z_{v}-p_{y_{v}} / 2\right|+\left|z_{v}-z_{v^{\prime}}\right|+\left|p_{y_{v^{\prime}}} / 2-z_{v^{\prime}}\right| \\
& \leq \sqrt{2 n \log \left(m k n^{2}\right)}+\left|z_{v}-z_{v^{\prime}}\right|
\end{aligned}
$$

which implies that $\left|z_{v}-z_{v^{\prime}}\right| \geq g / 2-\sqrt{2 n \log \left(m k n^{2}\right)}>\sqrt{2 n \log \left(m n^{3}\right)}$. Hence $\left(v, v^{\prime}\right) \notin E$.

The above two properties reveal that each connected component can be identified with a single $1 u \in[k]$ and the component contains exactly the received 1 s corresponding to that original one (formally $C_{u}=\left\{v: y_{v}=u\right\}$ ). From here we simply use the binomial estimator on each component. First observe that, by a Chernoff bound, with probability $1-k \exp (-m / 36)$, each 1 from the original string appears in at least a $1 / 3$-fraction of the traces, so that $\left|C_{u}\right| \geq m / 3$. Then apply the guarantee for the binomial mean estimator along with another union bound over the $k$ positions. Overall the failure probability is

$$
n^{-2}+k \exp (-m / 36)+2 k \exp \left(\frac{-m}{24 n^{2}}\right)
$$

which is at most $3 n^{-2}$ with $m \geq 24 n^{2} \log \left(2 k n^{2}\right)$. With this choice, we can tolerate $g=$ $O(\sqrt{n \log n})$.

\section{The recursion}

We now use the algorithm FindPositions in a recursive manner to estimate the parameters $p_{1}, \ldots, p_{k}$ even when the gap $g$ is much less than $\sqrt{n \log n}$. Define a series of threshold parameters, to be used in the $d^{\text {th }}$ level of recursion: 
Algorithm 2 Algorithm RecurGap.

Initialize: Traces $\mathcal{T}=\left\{\tilde{x}_{j}\right\}_{j=1}^{m}$, gap lower bound $g \geq c k \log ^{2}(n)$.

For each received 1 , create vertex $v$ decorated with $\left(z_{v}, t_{v}\right)$ where $z_{v} \in[n]$ is the position of the received 1 and $t_{v} \in[m]$ is the index of the trace.

Set $z_{v}^{(1)}=z_{v}, V_{1}=V$

for $d=1, \ldots, D$ : do

Create graph $G_{d}$ with vertices $V_{d}$ and with edges

$$
E_{1}=\left\{(v, w) \in V_{d}:\left|z_{v}^{(d)}-z_{w}^{(d)}\right| \leq \tau_{d} / 4\right\}
$$

Extract connected components $C_{1}^{(d)}, \ldots, C_{k_{d}}^{(d)}$ of $G_{d}$.

For each connected component $C_{i}^{(d)}$, extract subtraces $\left\{\tilde{x}_{j}^{(d, i)}\right\}_{j=1}^{m}$ where $\tilde{x}_{j}^{(d, i)}=\tilde{x}_{j}[\ell, r]$ and $\ell=\min \left\{z_{v}: v \in C_{i}^{(d)}, t_{v}=j\right\}$ and $r=\max \left\{z_{v}: v \in C_{i}^{(d)}, t_{v}=j\right\}$.

Define $L^{(d, i)}=\max _{j} \operatorname{len}\left(\tilde{x}_{j}^{(d, i)}\right)$. If

$$
\operatorname{len}\left(\tilde{x}_{j}^{(d, i)}\right) \leq L^{(d, i)}-\sqrt{2 L^{(d, i)} \log (k m n)}
$$

delete all vertices $v \in C_{i}^{(d)}$ with $t_{v}=j$. Let $V_{d+1}$ be all surviving vertices.

For $v \in C_{i}^{(d)} \cap V_{d+1}$, define $z_{v}^{(d+1)}=z_{v}-\min \left\{z_{v^{\prime}}: v^{\prime} \in C_{i}^{(d)}, t_{v^{\prime}}=t_{v}\right\}$. end for

$$
\begin{aligned}
\tau_{1} & =4 \sqrt{2 n \log (m n k)} ; \\
\tau_{d} & =80 \sqrt{k \tau_{i-1} \log (m n k)}, \quad d=2, \ldots, D
\end{aligned}
$$

where the total number of levels is $D$. Note that, $\tau_{d} \leq 80^{2} \cdot 4 \sqrt{2} \cdot k^{1-\frac{1}{2^{d-1}}} n^{\frac{1}{2^{d}}} \log ^{1-1 / 2^{d}}(n m k)$. In particular, if $D=O(\log \log n)$ then we have $\tau_{D}=O(k \log (n))$.

Recall that $V$ is the vertex set for the graph used above, where each vertex corresponds to a received 1 and is associated with an unknown original one $y_{v}$. Our main theorem of this section is the following.

- Theorem 15. Assume $g \geq 2 \tau_{D}$ for some $D \leq \log \log (n)$. Then except with probability $1-1 / n$, Algorithm 2 (RecurGap) with $D$ levels of recursion returns sets $S_{1}, \ldots, S_{k} \subset V$ such that

1. For all $u \in[k], S_{u} \subset\left\{v \in V: y_{v}=u\right\}$.

2. $\left|S_{u}\right| \geq m / \log ^{5}(n)$.

The theorem follows from the three lemmas stated earlier. Here we re-state the lemmas in detail and provide the proofs.

- Lemma 16 (Consistency). At level d let $V_{d, u}=\left\{v \in V_{d}, y_{v}=u\right\}$ for each $u \in[k]$. Then with probability $1-1 / n^{2}$, for each $d$ and $u$ there exists some component $C_{i}^{(d)}$ at level $d$ such that $V_{d, u} \subset C_{i}^{(d)}$.

Lemma 17 (Length Bound). At level d, the following holds with probability at least $1-1 / n^{2}$ : For every component $C_{i}^{(d)}$ at level $d$, we have $L^{(d, i)} \leq 2 k \tau_{d}$. Moreover if $U$ is a contiguous subsequence of $\{1, \ldots, k\}$ with $\bigcup_{u \in U} V_{d, u} \subset C_{i}^{(d)}$, then $\min _{u \in U} p_{u}-\max _{u \in U} p_{u} \leq 4 k \tau_{d}$. 
- Lemma 18 (Length Filter). Assume $m \geq n$. At level d, the following holds with probability at least $1-1 / n^{2}$ : For a component $C_{i}^{(d)}$ at level $d$, let $U$ be the maximal contiguous subsequence of $\{1, \ldots, k\}$ such that $\bigcup_{u \in U} V_{d, u} \subset C_{i}^{(d)}$. Define $u_{L}=\arg \min _{u \in U} p_{u}$ and $u_{R}=\arg \max _{u \in U} p_{u}$. Then for any $v \in C_{i}^{(d)}$, if $u_{L}$ and $u_{R}$ are present in $t_{v}$, then $v$ survives to round $d+1$, that is $v \in V_{d+1}$. Moreover, for any $v \in V_{d+1}$, let $p_{\min }(v, U)$ denote the original position of the first 1 from $U$ that is also in the trace $t_{v}$. Then we have $p_{\min }(v, u)-p_{u_{L}} \leq 8 \sqrt{k \tau_{d} \log (n m k)}$.

The proofs of the lemmas are all-intertwined. In the induction step we will assume that all lemmas hold at the previous level of the recursion. Throughout we repeatedly take union bound over all $m$ traces and all up-to- $k$ components, and set the failure probability for each event to be $1 / n^{2}$. In applications of Hoeffding's inequality, this produces a $2 \log (n m k)$ term inside the square root.

Proof of Lemma 17. We proceed by induction. For the base case, by Hoeffding's inequality, we know that for all $v \in V_{1}$ we have

$$
\left|z_{v}-p_{y_{v}} / 2\right| \leq \sqrt{n \log (m k n)}=\tau_{1} / 8
$$

except with probability $n^{-2}$. This means that the position corresponding to a single original $1 u$ can span at most $\tau_{1} / 4$ positions. If two such regions are merged into a single connected component, then the distance between the regions is at most $\tau_{1}$, by construction. Since there are most $k$ such regions, the total length is at most $(k-1) \tau_{1}+k \tau_{1} / 4 \leq 2 k \tau_{1}$. The second claim follows from the concentration statement.

For the induction step, assume that the connected components at level $d-1$ have length at most $2 k \tau_{d-1}$. Fix a connected component $C_{i}^{(d-1)}$ and let $u_{i, 1}^{(d-1)}$ denote the left-most original 1 present in $C_{i}^{(d-1)}\left(u_{i, 1}^{(d-1)}=\min \left\{y_{v}: v \in C_{i}^{(d-1)}\right\}\right)$. By another application of Hoeffding's inequality and using the error guarantee in Lemma 18, we have that

$$
\begin{aligned}
& \left|z_{v}^{(d-1)}-\left(p_{y_{v}}-p_{u_{i, 1}^{(d-1)}}\right) / 2\right| \\
& \leq\left|z_{v}^{(d-1)}-\left(p_{y_{v}}-p_{\min }\left(v, U_{i}^{(d-1)}\right)\right) / 2\right|+\left|p_{\min }\left(v, U_{i}^{(d-1)}\right)-p_{u_{i, 1}^{(d-1)}}\right| / 2 \\
& \leq \sqrt{2 k \tau_{d-1} \log (m k n)}+8 \sqrt{k \tau_{d-1} \log (m k n)} \leq \tau_{d} / 8
\end{aligned}
$$

except with probability $n^{-2}$. From here, the same argument as in the base case yields the claim.

Proof of Lemma 18. We have two conditions to verify. Fix a component $C_{i}^{(d)}$ at level $d$ with maximal contiguous subsequence $U \subset[k]$ and recall the definitions $u_{L}=\arg \min _{u \in U} p_{u}$ and $u_{R}=\arg \max _{u \in U} p_{u}$. By another concentration bound, we know that

$$
\forall j: \operatorname{len}\left(\tilde{x}_{j}^{(d, i)}\right) \leq\left(p_{u_{R}}-p_{u_{L}}\right) / 2+\sqrt{\left(p_{u_{R}}-p_{u_{L}}\right) \log (m n k)}
$$

with probability $1-n^{2}$. This reveals that:

$$
L^{(d, i)} \leq\left(p_{u_{R}}-p_{u_{L}}\right) / 2+\sqrt{\left(p_{u_{R}}-p_{u_{L}}\right) \log (m n k)}
$$

Moreover, for any trace $j$ that contains $u_{R}, u_{L}$ the tail bound is two-sided:

$$
\forall j \text { s.t. } u_{L}, u_{R} \in \tilde{x}_{j}^{(d, i)}:\left|\operatorname{len}\left(\tilde{x}_{j}^{(d, i)}\right)-\left(p_{u_{R}}-p_{u_{L}}\right) / 2\right| \leq \sqrt{\left(p_{u_{R}}-p_{u_{L}}\right) \log (m n k)} \text {. }
$$


Note that we also have $L^{(d, i)} \geq\left(p_{u_{R}}-p_{u_{L}}\right) / 2$ with overwhelming probability as:

$$
\begin{aligned}
& \operatorname{Pr}\left[\forall j: \operatorname{len}\left(\tilde{x}_{j}^{(d, i)}\right) \leq\left(p_{u_{R}}-p_{u_{L}}\right) / 2\right] \\
& \leq \prod_{j=1}^{m} \operatorname{Pr}\left[\operatorname{len}\left(\tilde{x}_{j}^{(d, i)}\right) \leq\left(p_{u_{R}}-p_{u_{L}}\right) / 2 \mid u_{R}, u_{L}\right] \cdot \operatorname{Pr}\left[u_{R}, u_{L}\right] \\
& \leq\left(\frac{1}{2} \cdot \frac{1}{4}\right)^{m}=2^{-3 m}
\end{aligned}
$$

Here we are using the symmetry of the binomial distribution. Thus, with $m \geq n$, the failure probability here is $\exp (-\Omega(n))$ ), which is negligible.

Using the upper bound on $L^{(d, i)}$ reveals that $\tilde{x}_{j}^{(d, i)}$ survives, since

$$
\begin{aligned}
\operatorname{len}\left(\tilde{x}_{j}^{(d, i)}\right) & \geq\left(p_{u_{R}}-p_{u_{L}}\right) / 2-\sqrt{\left(p_{u_{R}}-p_{u_{L}}\right) \log (m n k)} \\
& \geq L^{(d, i)}-2 \sqrt{\left(p_{u_{R}}-p_{u_{L}}\right) \log (m n k)} \geq L^{(d, i)}-2 \sqrt{2 L^{(d, i)} \log (m n k)} .
\end{aligned}
$$

For the second condition, assume that some trace $j$ survives but does not contain $u_{L}$. Let $u_{\min }=\arg \min \left\{y_{v}: v \in C_{i}^{(d)}, t_{v}=j\right\}$ denote the first original 1 in this trace that belongs to $C_{i}^{(d)}$ s block (By definition $p_{u_{\min }}=p_{\min }(v, U)$ for each $v: t_{v}=j$ ). Then we know that

$$
\begin{aligned}
\operatorname{len}\left(\tilde{x}_{j}^{(d, i)}\right) & \leq\left(p_{u_{R}}-p_{u_{\min }}\right) / 2+\sqrt{\left(p_{u_{R}}-p_{u_{\min }}\right) \log (n m k)} \\
& \leq\left(p_{u_{R}}-p_{u_{\min }}\right) / 2+\sqrt{2 L^{(d, i)} \log (n m k)}
\end{aligned}
$$

but since $\tilde{x}_{j}^{(d, i)}$ passed through the length filter, we also have a lower bound on its length, and so we get that

$$
p_{u_{\min }}-p_{u_{L}} \leq 4 \sqrt{2 L^{(d, i)} \log (n m k)} \leq 8 \sqrt{k \tau_{d} \log (n m k)}
$$

where the last inequality follows from Lemma 17.

Proof of Lemma 16. The proof here follows the discussion in the previous subsection. Fix a component $C_{i}^{(d-1)}$ with corresponding block $U_{i}^{(d-1)} \subset[k]$ at level $d-1$ and assume that all three lemmas apply for all previous levels. For a subtrace $x_{j}^{(d-1, i)}$ in this component observe and recall the definition $u_{i, 1}^{(d-1)}=\min \left\{y_{v}: v \in C_{i}^{(d-1)}\right\}$ and $p_{\min }\left(v, U_{i}^{(d-1)}\right)$, which is the position of the first 1 in $U_{i}^{(d-1)}$ that appears in trace $t_{v}=j$. Since the length of the subtrace is at most $2 k \tau_{d-1}$ by Lemma 17 we get that

$$
\begin{aligned}
& \left|z_{v}^{(d-1)}-\left(p_{t_{v}}-p_{u_{i, 1}^{(d-1)}}\right) / 2\right| \\
& \leq\left|z_{v}^{(d-1)}-\left(p_{t_{v}}-p_{\min }\left(v, U_{i}^{(d-1)}\right)\right) / 2\right|+\left|p_{\min }\left(v, U_{i}^{(d-1)}\right)-p_{u_{i, 1}^{(d-1)}}\right| / 2 \\
& \leq \sqrt{2 k \tau_{d-1} \log (m n k)}+8 \sqrt{k \tau_{d-1} \log (m k n)}=\tau_{d} / 8 .
\end{aligned}
$$

Here the last inequality uses Hoeffding's bound along with Lemma 18 at level $d-1$. This implies that the clustering at level $d$ is consistent.

Proof of Theorem 15. First take a union bound over $D \leq \log \log n$ applications of the three lemmas, so that the total failure probability is $c D / n^{2} \leq 1 / n$. From now, assume that the events in the three lemmas all hold for all levels. In particular, this implies that the components $C_{i}^{(D)}$ are consistent. We must verify that the clusters are pure and then track how many vertices remain. 


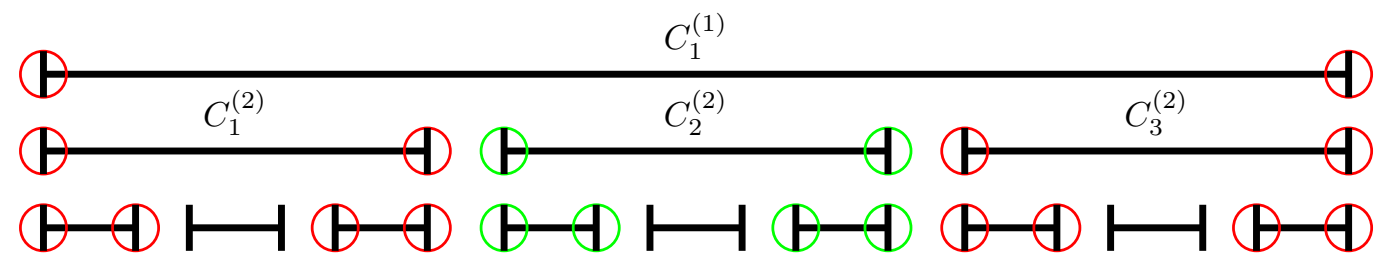

Figure 1 De-biasing of traces. The figure displays the regions of the original string $x$ that correspond to each connected component found in the algorithm. The end-points of each component correspond to $1 \mathrm{~s}$ in the original string. To de-bias the length filter for component $C_{1}^{(1)}$ at level 1, we identify and retain only the traces that contain all of the 1s colored red above. Then, to de-bias the length filter at $C_{2}^{(2)}$ at level 2, we identify and retain only the traces that contain all of the green 1 s.

For the first claim, let us revisit the proof of Lemma 16. If two vertices, say $v, v^{\prime}$, in a component at level $D-1$ corresponded to different $1 \mathrm{~s}$, say $u, u^{\prime}$ then by the gap condition, we know that $\left|p_{u}-p_{u^{\prime}}\right| \geq g$. On the other hand, we know that (2) holds, and we will use this to prove that no edge appears between these vertices. We have that

$$
\left|z_{v}-z_{v^{\prime}}\right| \geq \tau_{D} / 8+\tau_{D} / 8+\left|p_{t_{v}}-p_{t_{v}^{\prime}}\right| / 2 \geq \tau_{D} / 4+g / 2,
$$

and so, if $g / 2 \geq \tau_{D}$, then the two vertices will not share an edge. The argument applies for all pairs and hence the clusters at level $D$ are pure, which establishes the first claim in the Theorem 15.

For the second claim, note that by Lemma 18, for every component at every level, if a trace contains the two endpoints of that component, then it will survive the filter. Hence, in every filtering step we expect to retain $1 / 4$ of the subtraces passing through, and, by a Chernoff bound, we will retain $1 / 5$ of the subtraces except with $\exp (-\Omega(n))$, provided $m \geq n$. Since we perform $D=\log \log n$ levels, we retain $m / 5^{\log \log n}=m / \log ^{5}(n)$ traces in each cluster with high probability.

\section{Removing Bias: The reverse recursion}

Now that we have isolated the vertices into pure clusters, we need to work our way up through the recursion to remove biases introduced by the hierarchical clustering. For any component $C_{i}^{(D-1)}$ corresponding to block $U_{i}^{(D-1)} \subset[k]$ at level $D-1$, since the components at level $D$ are pure, we can identify exactly the subtraces that contain the first and last 1 in the block. We throw away all other traces, which de-biases the length filter at level $D-1$.

Unfortunately for a component $C_{i}^{(d-1)}$ corresponding to a block $U_{i}^{(d-1)}$ at level $d-1$, we cannot identify exactly the subtraces that contain the exactly the first and last 1 in the block. However, we know that $C_{i}^{(d-1)}$ is further refined into sub-components $\left\{C_{i^{\prime}}^{(d)}\right\}$ at level $d$, and by induction we can identify all the traces that contain the left-most and right-most 1 in the left-most and right-most sub-components. We identify all such traces and eliminate the rest to debias the length filter at level $d-1$. See Figure 1 for an illustration.

To debias this length filter, we filter based on the presence of two 1 s at level $d-1$ (just the end points), and two futher 1s at level $d$ (the inner endpoints of the first and last sub-components), four further 1 s at $d+1$, and so on. So, just to debias the length filter at level $d-1$ we require $2^{D-(d-1)} 1$ s to be present. Since we must debias all length filters above a particular component, we require the presence of $\sum_{d=1}^{D-1} 2^{D-d} \leq 2^{D} \leq \log _{2}(n) 1 \mathrm{~s}$. The probability of all $\log _{2}(n)$ of these 1 s appearing is $1 / n$ and by Chernoff bound, with high probability at least $m / 2 n$ of our traces will contain all of these $1 \mathrm{~s}$. 
For any $1, u$, in the original string, let $S$ denote the subset of $\log _{2}(n) 1 \mathrm{~s}$, whose presence we require to debias the length filters above the pure component containing $u$. After the debiasing step, the remaining vertices in the component containing $u$ have $z_{v}$ values distributed as

$$
z_{v} \sim \operatorname{Bin}\left(p_{u}-1-\left|S_{L}\right|, 1 / 2\right)+\left(\left|S_{L}\right|+1\right)
$$

where $\left|S_{L}\right|$ is the number of $1 \mathrm{~s}$ in $|S|$ that appear before $u$ in the sequence, and the final 1 is due to the presence of $u$. Using the binomial mean estimator, we can therefore estimate $p_{u}$ with probability $1-1 / n$, provided $m / n \geq n^{2} \log (n)$. Thus, $\operatorname{poly}(n)$ traces suffice to recover all $p_{u}$ values, provided that $g>\tau_{D}$ and $D=\log _{2} \log _{2} n$. This proves Theorem 2 .

\section{$4 \quad$ Reconstructing Arbitrary Matrices}

Recall that in the matrix reconstruction problem, we are given samples of a matrix $X \in$ $\{0,1\}^{\sqrt{n} \times \sqrt{n}}$ passed through a matrix deletion channel, which deletes each row and each column independently with probability $p=1-q$. In this section we prove Theorem 4 .

- Theorem (Restatement of Theorem 4). For matrix reconstruction, $\exp \left(O\left(n^{1 / 4} \sqrt{p \log n} / q\right)\right)$ traces suffice to recover an arbitrary matrix $X \in\{0,1\}^{\sqrt{n} \times \sqrt{n}}$, where $p$ is the deletion probability and $q=1-p$.

The bulk of the proof involves designing a procedure to test between two matrices $X$ and $Y$. This test is based on identifying a particular received entry where the traces must differ significantly, and to show this, we analyze a certain bivariate Littlewood polynomial, which is the bulk of the proof. Equipped with this test, we can apply a union bound and simply search over all pairs of matrices to recover the string.

For a matrix $X \in\{0,1\}^{\sqrt{n} \times \sqrt{n}}$, let $\tilde{X}$ denote a matrix trace. Let us denote the $(i, j)^{\text {th }}$ entry of the matrix as $X_{i, j}, i, j=0,1, \ldots, \sqrt{n}-1$, an indexing protocol we adhere to for every matrix. For two complex numbers $w_{1}, w_{2} \in \mathbb{C}$, observe that

$$
\begin{aligned}
\mathbb{E}\left[\sum_{i, j=0}^{\sqrt{n}-1} \tilde{X}_{i, j} w_{1}^{i} w_{2}^{j}\right] & =q^{2} \sum_{i, j} w_{1}^{i} w_{2}^{j} \sum_{k_{i} \geq i, k_{j} \geq j} X_{k_{i}, k_{j}}\left(\begin{array}{c}
k_{i} \\
i
\end{array}\right)\left(\begin{array}{c}
k_{j} \\
j
\end{array}\right) p^{k_{i}-i} q^{i} p^{k_{j}-j} q^{j} \\
& =q^{2} \sum_{k_{1}, k_{2}=0}^{\sqrt{n}-1} X_{k_{1}, k_{2}}\left(q w_{1}+p\right)^{k_{1}}\left(q w_{2}+p\right)^{k_{2}}
\end{aligned}
$$

Thus, for two matrices $X, Y$, we have

$$
\begin{aligned}
\frac{1}{q^{2}} \mathbb{E}\left[\sum_{i, j=0}^{\sqrt{n}-1}\left(\tilde{X}_{i, j}-\tilde{Y}_{i, j}\right) w_{1}^{i} w_{2}^{j}\right] & =\sum_{k_{1}, k_{2}=0}^{\sqrt{n}-1}\left(X_{k_{1}, k_{2}}-Y_{k_{1}, k_{2}}\right)\left(q w_{1}+p\right)^{k_{1}}\left(q w_{2}+p\right)^{k_{2}} \\
& \triangleq A\left(z_{1}, z_{2}\right)
\end{aligned}
$$

where we are rebinding $z_{1}=q w_{1}+p$ and $z_{2}=q w_{2}+p$. Observe that $A\left(z_{1}, z_{2}\right)$ is a bivariate Littlewood polynomial; all coefficients are in $\{-1,0,1\}$, and the degree is $\sqrt{n}$. For such polynomials, we have the following estimate, which extends a result due of Borwein and Erdélyi [6] for univariate polynomials.

Lemma 19. Let $f\left(z_{1}, z_{2}\right)$ be non-zero degree $n$ Littlewood polynomial. Then,

$$
\left|f\left(z_{1}^{\star}, z_{2}^{\star}\right)\right| \geq \exp \left(-C_{1} L^{2} \log n\right)
$$

for some $z_{1}^{\star}=\exp \left(i \theta_{1}\right), z_{2}^{\star}=\exp \left(i \theta_{2}\right)$ where $\left|\theta_{1}\right|,\left|\theta_{2}\right| \leq \pi / L$, and $C_{1}$ is a universal constant. 
Proof. Fix $L>0$ and define the polynomial

$$
F\left(z_{1}, z_{2}\right)=\prod_{1 \leq a \leq L, 1 \leq b \leq L} f\left(z_{1} e^{\pi i a / L}, z_{2} e^{\pi i b / L}\right) .
$$

We first show that there exists $z_{1}^{\star}, z_{2}^{\star}$ on the unit disk such that $F\left(z_{1}^{\star}, z_{2}^{\star}\right) \geq 1$. This follows from an iterated application of the maximum modulus principle. First factorize $F\left(z_{1}, z_{2}\right)=z_{2}^{k} G\left(z_{1}, z_{2}\right)$ where $k$ is chosen such that $G\left(z_{1}, z_{2}\right)$ has no common factors of $z_{2}$. Since $F$ has non-zero coefficients, this implies that $G\left(z_{1}, 0\right)$ is a non-zero univariate polynomial. Further factorize $G\left(z_{1}, 0\right)=z_{1}^{\ell} H\left(z_{1}\right)$ so that terms in $H$ have no common factors of $z_{1}$. $H$ is also a Littlewood polynomial and moreover it has non-zero leading term, so that $|H(0)| \geq 1$. Thus by the maximum modulus principle:

$$
\left|F\left(z_{1}^{\star}, z_{2}^{\star}\right)\right|=\left|G\left(z_{1}^{\star}, z_{2}^{\star}\right)\right| \geq\left|G\left(z_{1}^{\star}, 0\right)\right| \geq\left|H\left(z_{1}^{\star}\right)\right| \geq|H(0)| \geq 1 .
$$

Now, for any $a, b \in\{1, \ldots, L\}$ we have

$$
1 \leq\left|F\left(z_{1}^{\star}, z_{2}^{\star}\right)\right| \leq\left|f\left(\left(z_{1}^{\star}\right)^{\pi i a / L},\left(z_{2}^{\star}\right)^{\pi i b / L}\right)\right| \cdot n^{\left(L^{2}-1\right)},
$$

where we are using the fact that $\left|f\left(z_{1}, z_{2}\right)\right| \leq n$. This proves the lemma, since we may choose $a$ such that $\left(z_{1}^{\star}\right)^{\pi i a / L}=\exp (i \theta)$ for $|\theta| \leq \pi / L$.

Let $\gamma_{L}=\left\{e^{i \theta}:|\theta| \leq \pi / L\right\}$ denote the arc specified in Lemma 19. For any $z_{1} \in \gamma_{L}$, Nazarov and Peres [26] provide the following estimate for the modulus of $w_{1}=\left(z_{1}-p\right) / q$ :

$$
\forall z \in \gamma_{L}:|(z-p) / q| \leq \exp \left(C_{2} p /(L q)^{2}\right) .
$$

Using these two estimates, we may sandwich $\left|A\left(z_{1}, z_{2}\right)\right|$ by

$$
\exp \left(-C_{1} L^{2} \log n\right) \leq \max _{z_{1}, z_{2} \in \gamma_{L}}\left|A\left(z_{1}, z_{2}\right)\right| \leq \frac{\exp \left(C^{\prime} p \sqrt{n} /(L q)^{2}\right)}{q^{2}} \sum_{i j}\left|\mathbb{E}\left[\tilde{X}_{i j}-\tilde{Y}_{i j}\right]\right|
$$

This implies that there exists some coordinate $(i, j)$ such that

$$
\left|\mathbb{E}\left[\tilde{X}_{i j}-\tilde{Y}_{i j}\right]\right| \geq \frac{q^{2}}{n} \exp \left(-C_{1} L^{2} \log n-\frac{C^{\prime} p \sqrt{n}}{L^{2} q^{2}}\right) \geq \frac{q^{2}}{n} \exp \left(-C \frac{n^{1 / 4} \sqrt{p \log n}}{q}\right),
$$

where the second inequality follows by optimizing for $L$.

The remainder of the proof follows the argument of [26]: Since we have witnessed significant separation between the traces received from $X$ and those received from $Y$, we can test between these cases with $\exp \left(O\left(n^{1 / 4} \sqrt{\log n}\right)\right)$ samples (via a simple Chernoff bound). Since we do not know which of the $2^{n}$ traces is the truth, we actually test between all pairs, where the test has no guarantee if neither matrix is the truth. However, via a union bound, the true matrix will beat every other in these tests and this only introduces a $\operatorname{poly}(n)$ factor in the sample complexity.

\section{$5 \quad$ Reconstructing Random Matrices}

In this section, we prove Theorem 5: $O(\log n)$ traces suffice to reconstruct a random $\sqrt{n} \times \sqrt{n}$ matrix with high probability for any constant deletion probability $p<1$. This is optimal since $\Omega(\log n)$ traces are necessary to just ensure that every bit appears in a least one trace.

Our result is proved in two steps. We first design an oracle that allows us to identify when two rows (or two columns) in different matrix traces correspond to the same row (resp. column) of the original matrix. We then use this oracle to identify which rows and columns of the original matrix have been deleted to generate each trace. This allows us to identify the original position of each bit in each trace. Hence, as long as each bit is preserved in at least one trace (and $O(\log n)$ traces is sufficient to ensure this with high probability), we can reconstruct the entire original matrix. 
Oracle for Identifying Corresponding Rows/Columns. We will first design an oracle that given two strings $t$ and $t^{\prime}$ distinguishes, for any constant $q>0$, with high probability between the cases:

Case 1: $t$ and $t^{\prime}$ are traces generated by the deletion channel with preservation probability $q$ from the same random string $x \in_{R}\{0,1\}^{\sqrt{n}}$

Case 2: $t$ and $t^{\prime}$ are traces generated by the deletion channel with preservation probability $q$ from independent random strings $x, y \in R\{0,1\}^{\sqrt{n}}$

It $t$ and $t^{\prime}$ are two rows (or two columns) from two different matrix traces, then this test determines whether $t$ and $t^{\prime}$ correspond to the same or different row (resp. column) of the original matrix. In Section 5.1, we show how to perform this test with failure probability at most $1 / n^{10}$. In fact, the failure probability can be made exponentially small but a polynomially small failure probability will be sufficient for our purposes.

Using the Oracle for Reconstruction. Given $m=\Theta(\log n)$ traces we can ensure that every bit of $X$ appears in at least one of the matrix traces with high probability. We then use this oracle to associate each row in each trace with the rows in other traces that are subsequences of the same original row. This requires at $\operatorname{most}\left(\begin{array}{c}m \sqrt{n} \\ 2\end{array}\right) \leq(m \sqrt{n})^{2}$ applications of the oracle and so, by the union bound, this can performed with failure probability at most $(m \sqrt{n})^{2} / n^{10} \leq 1 / n^{8}$ where the inequality applies for sufficiently large $n$.

After using the oracle to identify corresponding rows amongst the different traces we group all the rows of the traces into $\sqrt{n}$ groups $G_{1}, \ldots, G_{\sqrt{n}}$ where the expected size of each group is $m q$. We next infer which group corresponds to the $i^{\text {th }}$ row of $X$ for each $i \in[\sqrt{n}]$. Let $f$ be the bijection between groups and $[\sqrt{n}]$ that we are trying to learn, i.e., $f(j)=i$ if the $j^{\text {th }}$ group corresponds to the $i^{\text {th }}$ row of $X$. If suffices to determine whether $f(j)<f\left(j^{\prime}\right)$ or $f(j)>f\left(j^{\prime}\right)$ for each pair $j \neq j^{\prime}$. If there exists a matrix trace $\tilde{X}$ that includes a row in $G_{j}$ and a row in $G_{j^{\prime}}$ then we can infer the relative ordering of $f(j)$ and $f\left(j^{\prime}\right)$ based on whether the row from $G_{j}$ appears higher or lower in $\tilde{X}$ than the row in $G_{j^{\prime}}$. The probability there exists such a trace is $1-\left(1-q^{2}\right)^{m} \geq 1-1 / \operatorname{poly}(n)$ and we can learn the bijection $f$ with high probability.

We also perform an analogous process with columns. After both rows and columns have been processed, we know exactly which rows and columns were deleted to form each trace, which reveals the original position of each received bit in each trace. Given that every bit of $X$ appeared in at least some trace, this suffices to reconstruct $X$, proving Theorem 5 .

- Theorem (Restatement of Theorem 5). For any constant deletion probability $p<1, O(\log n)$ traces are sufficient to reconstruct a random $X \in\{0,1\}^{\sqrt{n}} \times \sqrt{n}$.

\subsection{Oracle: Testing whether two traces come from same random string}

Define $S_{i}=\{2 w i+j: j=0, \ldots, w-1\}$ to be a contiguous subsets of size

$$
w=100 n^{1 / 4} \sqrt{1 / q \cdot \log n} .
$$

Note that there are size $w$ gaps between each $S_{i}$ and $S_{i+1}$, i.e., $w$ elements that are both larger than $S_{i}$ and smaller than $S_{i+1}$. This will later help us argue that the bits in positions $S_{i}$ and $S_{i+1}$ in different traces are independent. Given a traces $t, t^{\prime}$, define the three quantities: $X_{i}=\sum_{j \in S_{i}} t_{j}, Y_{i}=\sum_{j \in S_{i}} t_{j}^{\prime}$ and $Z_{i}=\left(X_{i}-Y_{i}\right)^{2}$. We will show that by considering $Z_{0}, Z_{1}, Z_{2}, \ldots$ we can determine whether $t$ and $t^{\prime}$ are traces of the same original string or traces of two different random strings. 
The basic idea is that if $t$ and $t^{\prime}$ are generated by the same string, many of the bits summed to construct $X_{i}$ and the bits summed to construct $Y_{i}$ will correspond to the same bits of the original string; hence $Z_{i}$ will be smaller than it would be if $t$ and $t^{\prime}$ were generated from two independent random strings. To make this precise, we need to introduce some additional notation.

- Definition 20. For $A \subset\{0,1,2, \ldots\}$, let $R_{t}(A)$ be the indices of the bits in the transmitted string that landed in positions $A$ in trace $t$. Similarly define $R_{t^{\prime}}(A)$. For example, if bits in position 0 and 2 were deleted during the transmission of then $R_{t}(\{0,1,2\})=\{1,3,4\}$.

The next lemma quantifies the overlap between $R_{t}\left(S_{i}\right)$ and $R_{t^{\prime}}\left(S_{i}\right)$.

- Lemma 21 (Deletion Patterns). With high probability over the randomness of the deletion channel,

$$
\forall i,\left|R_{t}\left(S_{i}\right) \cap R_{t^{\prime}}\left(S_{i}\right)\right| \geq q w / 2 \quad \text { and } \quad \forall i \neq j,\left|R_{t}\left(S_{i}\right) \cap R_{t^{\prime}}\left(S_{j}\right)\right|=0 .
$$

Note that conditioned on the second property, each $Z_{i}$ is independent.

Proof. First note that by the Chernoff bound, for each $j \in[\sqrt{n}]$, the $j^{\text {th }}$ bit of the original sequence appears in position $q j \pm r$ where $r=5 n^{1 / 4} \sqrt{q \log n}$ with high probability. The second part of the lemma follows since $r=w q / 20<w / 20$ and therefore, with high probability, any bit in the original string will not appear in $S_{\alpha}$ in one trace and $S_{\beta}$ in another for $\alpha \neq \beta$ because there was a size $w$ gap between $S_{\alpha}$ and $S_{\beta}$.

For the first part of the lemma, for each $S_{i}$, define

$$
S_{i}^{\prime}=\{2 w i / q+r / q, 2 w i / q+r+1, \ldots,(2 w i+w-1) / q-r / q\}
$$

By the Chernoff Bound, with high probability the $w / q-2 r / q>0.9 w / q$ bits in $S_{i}^{\prime}$ positions in the original string arrive in positions $S_{i}$ in the trace. Also with high probability, $0.9 q^{2}\left|S_{i}^{\prime}\right|$ of the bits in $S_{i}^{\prime}$ are transmitted in the generation of both $t$ and $t^{\prime}$. Hence, $\left|R_{t}\left(S_{i}\right) \cap R_{t^{\prime}}\left(S_{i}\right)\right| \geq$ $0.9 w / q \cdot 0.9 q^{2}>q w / 2$ as required.

We are now ready to argue that the values $Z_{0}, Z_{1}, \ldots$ are sufficient to determine whether or not $t$ and $t^{\prime}$ are generated from the same random string.

- Theorem 22. Let $z_{j}=\sum_{i=0}^{g-1} Z_{j g+i}$ for $g=96 / q^{2}$ and $D=\operatorname{median}\left(z_{0}, z_{1}, z_{2}, \ldots, z_{\Theta(\log n)}\right)$. Case 1: If $t$ and $t^{\prime}$ are generated from the same string, then $\operatorname{Pr}[D<(1-q / 4) g w / 2] \geq$ $1-1 / n^{10}$

Case 2: If $t$ and $t^{\prime}$ are generated from different strings, then $\operatorname{Pr}[D \geq(1-q / 4) g w / 2] \geq$ $1-1 / n^{10}$.

Proof. Throughout the proof we condition on the equations in Lemma 21 being satisfied. Note that this event is a function of the randomness of the deletion channel rather than the randomness of the strings being transmitted over the deletion channel.

First, suppose $t$ and $t^{\prime}$ are generated from different strings. Then $Z_{i}$ has the same distribution as the variable $C$ in Lemma 23 when $r$ is set to $w$. Hence, $\mathbb{E}\left[z_{j}\right]=g w / 2$ and $\operatorname{var}\left(z_{j}\right) \leq g w^{2} / 2$. Therefore,

$$
\operatorname{Pr}\left[z_{j}<(1-q / 4) g w / 2\right] \leq \operatorname{Pr}\left[\left|z_{j}-\mathbb{E}\left[z_{j}\right]\right| \geq(q / 4) g w / 2\right] \leq \frac{\operatorname{var}\left(z_{j}\right)}{\mathbb{E}\left[z_{j}\right]^{2} \cdot q^{2} / 16} \leq \frac{2}{g q^{2} / 16}=1 / 3 .
$$

Therefore, by the Chernoff bound, $D \geq(1-q / 4) g w / 2$ with probability at least $1-1 / n^{10}$. 
Now, suppose $t$ and $t^{\prime}$ are generated from the same string. Then, $Z_{i}$ has the same distribution as $C$ in Lemma 23 for some $r \leq w-q w / 2$. Hence, $\mathbb{E}\left[z_{j}\right]=g r / 2$ and $\operatorname{var}\left(z_{j}\right) \leq$ $g r^{2} / 2$. Therefore,

$$
\operatorname{Pr}\left[z_{j} \geq(1-q / 4) g w / 2\right] \leq \operatorname{Pr}\left[\left|z_{j}-\mathbb{E}\left[z_{j}\right]\right| \geq(q / 4) g w / 2\right] \leq \frac{\operatorname{var}\left(z_{j}\right)}{\mathbb{E}\left[z_{j}\right]^{2} \cdot q^{2} / 16} \leq \frac{2}{g q^{2} / 16}=1 / 3 .
$$

Therefore, by the Chernoff bound, $D<(1-q / 4) g w / 2$ with probability at least $1-1 / n^{10}$.

Lemma 23. Let $A \sim \operatorname{Bin}(h, 1 / 2)$ and $B \sim \operatorname{Bin}(h, 1 / 2)$ be independent and $C=(A-B)^{2}$. Then,

$$
\mathbb{E}[C]=h / 2 \quad \text { and } \quad \operatorname{var}[C] \leq m^{2} / 2 .
$$

Proof. The result follows by direct calculation:

$$
\mathbb{E}\left[(A-B)^{2}\right]=\mathbb{E}\left[A^{2}\right]+\mathbb{E}\left[B^{2}\right]-2 \mathbb{E}[A] \mathbb{E}[B]=m(m+1) / 2-m^{2} / 2=m / 2
$$

and

$$
\operatorname{var}\left((A-B)^{2}\right)=\mathbb{E}\left[(A-B)^{4}\right]-(m / 2)^{2}=m / 2+6\left(\begin{array}{c}
m \\
2
\end{array}\right) / 4-m^{2} / 4=(2 m-1) m / 4 .
$$

\section{Bounded Hamming Distance}

In this section, we turn to the sparse testing problem. We show that is possible to distinguish between two strings $x$ and $y$ with Hamming distance $\Delta(x, y)<2 k$, given $\exp (O(k \log n))$ traces. This question is naturally related to sparse reconstruction, since the difference string $x-y \in\{-1,0,1\}^{n}$ is at most $2 k$ sparse, but distinguishing two strings from traces is also at the core of our analysis in Section 2, as well as the analysis of Nazarov and Peres [26] and De et al. [11]. In particular given a testing routine, reconstruction simply requires applying the union bound.

In the binary symmetric channel (where each bit is flipped independently with some probability), distinguishing between two strings is easier if the Hamming distance is larger, since the two strings are farther apart. However, it is unclear if this intuition carries over to the deletion channel. In particular, the number of traces required for testing is unlikely to even be monotonic in the Hamming distance; if the Hamming distance is odd, then $x$ and $y$ have different Hamming weight, and we can estimate the Hamming weight using just $O(n)$ traces.

Our analysis uses a combinatorial result about $k$-decks due to Krasikov and Roditty [23], along with an approach first used in McGregor et al. [24].

- Theorem 24 (Krasikov and Roditty [23]). The $k$-deck of a string is the multi-set of length $k$ subsequences. No two strings $x, y$ of length $n$ have the same $k$-deck if $\Delta(x, y)<2 k$.

Theorem 25. The $k$-deck of a binary string can be determined exactly with $\exp (O(k \log n))$ traces from the symmetric deletion channel assuming $p \leq 1-k / n$.

Proof. We argue that sampling $\exp (O(k \log n))$ length $k$-subsequence of a string is sufficient to reconstruct the $k$-deck with high probability. The result then follows because if $p \leq 1-k / n$, then with constant probability a trace generated by the deletion channel has length at least $k$ and hence we can take a random $k$ subsequence of such a trace as a random $k$ subsequence from $x$. 
Let $f_{u}$ be the number of times that $u \in\{0,1\}^{k}$ appears as a subsequence of $x$. Then, let $X_{u}$ be the number of times $u$ is generated if we sample $r=3 n^{k} \log n^{k}$ subsequences of length $k$ uniformly at random. $\mathbb{E}\left[X_{i}\right]=r f_{u} /\left(\begin{array}{l}n \\ k\end{array}\right)$ and by an application of the Chernoff bound.

$$
\operatorname{Pr}\left[\left|X_{u}\left(\begin{array}{l}
n \\
k
\end{array}\right) / r-f_{u}\right| \geq 1\right]=\operatorname{Pr}\left[\left|X_{u}-\mathbb{E}[X]\right| \geq r /\left(\begin{array}{l}
n \\
k
\end{array}\right)\right] \leq \exp \left(-\frac{f_{u}^{2} \cdot r f_{u}}{3\left(\begin{array}{l}
n \\
k
\end{array}\right)}\right) \leq 1 / n^{k}
$$

where the last line follows given $f_{u} \geq 1$ (if $f_{u}=0$ the bound is trivially true) and $r=$ $3 n^{k} \log n^{k}$. Hence, by taking the union bound over all $2^{k}$ sequences $u$, it follows that we can determine the frequency of all length $k$ subsequences with high probability.

Theorem 3 follows directly from Theorem 24 and Theorem 25 .

- Theorem (Restatement of Theorem 3). For all $x, y \in\{0,1\}^{n}$ such that $\Delta(x, y)<2 k$,

$$
m=\exp (O(k \log n))
$$

traces are sufficient to be distinguished between $x$ and $y$.

As noted earlier, if $\Delta(x, y)$ is odd then poly $(n)$ traces suffice. Also, regardless of the Hamming distance, if the location of the first and second positions (say $i$ and $j$ ) where $x$ and $y$ differs by at least $\Omega(\sqrt{n \log n})$ then it is easy to show that expected weight of the length $i / 2$ prefix of the traces differs by $\Omega(1 /$ poly $(n))$ and hence we can distinguish $x$ and $y$ with poly $(n)$ traces.

\section{References}

1 Dimitris Achlioptas and Frank McSherry. On spectral learning of mixtures of distributions. In Conference on Learning Theory, 2005.

2 Sanjeev Arora and Ravi Kannan. Learning mixtures of arbitrary gaussians. In Symposium on Theory of Computing, 2001.

3 Frank Ban, Xi Chen, Adam Frelich, Rocco A. Servedio, and Sandip Sinha. Beyond trace reconstruction: Population recovery from the deletion channel. arXiv, 2019. arXiv: 1904.05532.

4 Tugkan Batu, Sampath Kannan, Sanjeev Khanna, and Andrew McGregor. Reconstructing strings from random traces. In Symposium on Discrete Algorithms, 2004.

5 Mikhail Belkin and Kaushik Sinha. Polynomial learning of distribution families. In Foundations of Computer Science, 2010.

6 P. Borwein and T. Erdélyi. Littlewood-Type Problems on Subarcs of the Unit Circle. Indiana University Mathematics Journal, 1997.

7 Siu-On Chan, Ilias Diakonikolas, Rocco A Servedio, and Xiaorui Sun. Learning mixtures of structured distributions over discrete domains. In Symposium on Discrete Algorithms, 2013.

8 Mahdi Cheraghchi, Ryan Gabrys, Olgica Milenkovic, and João Ribeiro. Coded trace reconstruction. arXiv e-prints, page arXiv:1903.09992, March 2019. arXiv:1903.09992.

9 Sanjoy Dasgupta. Learning mixtures of Gaussians. In Foundations of Computer Science, 1999.

10 Sami Davies, Miklos Z. Racz, and Cyrus Rashtchian. Reconstructing Trees from Traces. arXiv e-prints, page arXiv:1902.05101, February 2019. arXiv:1902.05101.

11 Anindya De, Ryan O'Donnell, and Rocco A. Servedio. Optimal mean-based algorithms for trace reconstruction. In Symposium on Theory of Computing, 2017.

12 Jon Feldman, Ryan O'Donnell, and Rocco A Servedio. Learning mixtures of product distributions over discrete domains. SIAM Journal on Computing, 2008.

13 Anna C. Gilbert and Piotr Indyk. Sparse Recovery Using Sparse Matrices. Proceedings of the IEEE, 2010. 
14 Moritz Hardt and Eric Price. Tight bounds for learning a mixture of two gaussians. In Symposium on Theory of Computing, 2015.

15 Lisa Hartung, Nina Holden, and Yuval Peres. Trace reconstruction with varying deletion probabilities. In Workshop on Analytic Algorithmics and Combinatorics, 2018.

16 Nina Holden and Russell Lyons. Lower bounds for trace reconstruction. arXiv, 2018. arXiv: 1808.02336.

17 Nina Holden, Robin Pemantle, and Yuval Peres. Subpolynomial trace reconstruction for random strings and arbitrary deletion probability. In Conference On Learning Theory, COLT 2018, Stockholm, Sweden, 6-9 July 2018., pages 1799-1840, 2018.

18 Thomas Holenstein, Michael Mitzenmacher, Rina Panigrahy, and Udi Wieder. Trace reconstruction with constant deletion probability and related results. In Symposium on Discrete Algorithms, 2008.

19 Samuel B Hopkins and Jerry Li. Mixture models, robustness, and sum of squares proofs. In Symposium on Theory of Computing, 2018.

20 Adam Tauman Kalai, Ankur Moitra, and Gregory Valiant. Efficiently learning mixtures of two Gaussians. In Symposium on Theory of Computing, 2010.

21 Sampath Kannan and Andrew McGregor. More on Reconstructing Strings from Random Traces: Insertions and Deletions. In International Symposium on Information Theory, 2005.

22 Géza Kós, Péter Ligeti, and Péter Sziklai. Reconstruction of matrices from submatrices. Mathematics of Computation, 2009. doi:10.1090/S0025-5718-09-02210-8.

23 I. Krasikov and Y. Roditty. On a Reconstruction Problem for Sequences. Journal of Combinatorial Theory, Series A, 1997.

24 Andrew McGregor, Eric Price, and Sofya Vorotnikova. Trace Reconstruction Revisited. In European Symposium on Algorithms, 2014.

25 Ankur Moitra and Gregory Valiant. Settling the polynomial learnability of mixtures of gaussians. In Foundations of Computer Science, 2010.

26 Fedor Nazarov and Yuval Peres. Trace reconstruction with $\exp \left(O\left(n^{1 / 3}\right)\right.$ samples. In Symposium on Theory of Computing, 2017.

27 Yuval Peres and Alex Zhai. Average-Case Reconstruction for the Deletion Channel: Subpolynomially Many Traces Suffice. In Symposium on Foundations of Computer Science, 2017.

28 Krishnamurthy Viswanathan and Ram Swaminathan. Improved string reconstruction over insertion-deletion channels. In Symposium on Discrete Algorithms, 2008. 